\title{
Projet mystique, réseaux sociaux et mobilisation des ressources : le passage en Nouvelle-France de Marie de l'Incarnation en 1639
}

Françoise Deroy-Pineau et Paul Bernard

\section{(2) OpenEdition \\ Journals}

Édition électronique

URL : http://journals.openedition.org/assr/20187

DOI : $10.4000 /$ assr. 20187

ISSN : $1777-5825$

Éditeur

Éditions de l'EHESS

Édition imprimée

Date de publication : 1 avril 2001

Pagination : 61-91

ISBN : 2-222-96701-5

ISSN : 0335-5985

Référence électronique

Françoise Deroy-Pineau et Paul Bernard, « Projet mystique, réseaux sociaux et mobilisation des ressources : le passage en Nouvelle-France de Marie de l'Incarnation en 1639 », Archives de sciences sociales des religions [En ligne], 113 | janvier-mars 2001, mis en ligne le 19 août 2009, consulté le 30 avril 2019. URL : http://journals.openedition.org/assr/20187; DOI : 10.4000/assr.20187

Ce document a été généré automatiquement le 30 avril 2019.

(C) Archives de sciences sociales des religions 


\title{
Projet mystique, réseaux sociaux et mobilisation des ressources : le passage en Nouvelle-France de Marie de l'Incarnation en 1639
}

\author{
Françoise Deroy-Pineau et Paul Bernard
}

1 L'étude des réseaux sociaux permet d'appréhender la société d'une manière "mésosociale », en mettant en relief de façon inductive ce qui se déroule dans les interstices entre acteurs et systèmes institués. Là se situent les échanges de personne à personne animées d'un même projet ou de projets complémentaires, à courte ou longue échéance, économiques ou utopiques, prosaïques ou mystiques. Les études sur les réseaux mettent en exergue l'importance des liens, interpersonnels, ou entre différentes organisations et groupes; elles s'appliquent sur de nombreux terrains: diffusion des informations (Granovetter, 1973), emploi (Granovetter, 1974), mobilisation de ressources (Nan Lin, 1982a, 1982b, 1995), entraide (Degenne et Lebeaux, 1991) et vie de banlieue (Wellman, 1979, 1988 a, b, c, 1991), amitié (Bidart, 1991), associations sans but lucratif (Ferrand, 1982, 1991), structure intra ou inter-organisationnelle (Lazega, 1992, 1994, 1995 a, b). Elles montrent à l'œuvre des innovateurs (Nohria, 1992) et elles offrent des éclairages inattendus sur les processus sociaux, y compris sur des sujets appartenant à l'histoire, en particulier l'histoire religieuse (Meeks, 1983, à propos de Paul de Tarse ; Bertrand, 1985, 1988, à propos d'Ignace de Loyola).

2 C'est la perspective que nous avons nous-mêmes empruntée pour l'étude d'un sujet semblable, l'établissement des Ursulines à Québec en 1639, à l'initiative de Marie Guyart, dite Marie de l'Incarnation'. Cette Tourangelle sans argent ni pouvoir, portée par un courant mystique qui a fourni à certaines femmes l'occasion de se bâtir un destin exceptionnel ${ }^{2}$, a reconnu et mis à profit les caractéristiques des réseaux sociaux dans lesquels elle était insérée pour mobiliser d'importantes ressources humaines et matérielles et pour obtenir les autorisations exceptionnelles requises afin de mener à 
bien ce projet. Ce sont quelques caractéristiques structurelles de ces réseaux et les stratégies mises en oeuvre par Marie Guyart que nous décrirons ici; nous mettrons en relief l'engendrement mutuel de ces structures et de ces stratégies réticulaires, la production de l'acteur par le réseau et du réseau par l'acteur.

Il est bien entendu que nous ne réduisons ni l'œuvre, ni la personne et encore moins l'expérience de Marie de l'Incarnation à ces stratégies. On sait que Marie est d'abord une mystique étudiée comme telle par les théologiens de la spiritualité3. Nous nous limitons ici à essayer de faire ressortir un aspect bien particulier de l'incarnation de Marie dans le tissu social de son époque. L'expérience mystique - au moins dans son cas -, loin d'être désincarnée, motive des projets défiant la raison pratique à courte vue en utilisant des moyens prosaïques qui n'ont rien à voir avec la magie.

4 Après une courte biographie de Marie Guyart et quelques indications sur la méthodologie qui nous a permis de dégager son réseau social, nous développerons successivement plusieurs propositions analytiques d'ordre structurel, conjoncturel ou de la stratégie politique: Marie Guyart occupe une position-pivot, à la jointure de plusieurs réseaux antérieurs à son action; elle est douée d'une rare compétence réticulaire, sachant reconnaître et actualiser des virtualités chez les gens qu'elle fréquente ou rejoint, selon la conjoncture; elle articule en séquence liens forts et faibles, personnes-relais; elle sait étanchéifier et diversifier ses réseaux en fonction des résultats qu'il faut atteindre.

\section{1 - Marie Guyart et son projet}

Marie Guyart, qui sera connue sous le nom de Marie de l'Incarnation comme religieuseursuline et pionnière de la Nouvelle-France, étonne tout autant comme mystique que comme femme d'action ${ }^{4}$. Née à Tours en France en 1599, elle traverse les trois-quarts du $\mathrm{XVII}^{\mathrm{e}}$ siècle avec une détermination soutenue par un même dessein: épouser le mouvement de son " moteur intérieur $»^{5}$ qui la conduit vers des territoires insoupçonnés tant au niveau sociologique que psychologique ou géographique. En effet, née dans une famille d'artisans boulangers, elle est imprégnée dès le berceau, comme la plupart des gens de son époque, par les pratiques catholiques. Ses parents la marient à dix-sept ans. Deux ans plus tard, elle est veuve et mère de Claude Martin, le fils à qui elle écrira une abondante correspondance. C'est alors qu'elle entame une démarche mystique et décourage tout prétendant au remariage. Elle mène alors parallèlement à ce cheminement exceptionnel - et mis à part quelques mois d'isolement passés à broder chez son père - une vie très active dans l'entreprise de transports de son beau-frère. Entrée au bas de l'échelle, elle en devient gérante, et s'implique dans la vie sociale de Tours.

6 Malgré le déchirement causé par la rupture du lien mère-fils, lorsque ce dernier atteint l'âge d'entrer au collège (douze ans), elle le confie à sa sœur, laisse les affaires de son beau-frère et se dirige sans dot chez les Ursulines, un nouvel ordre religieux chargé d'une vocation alors originale : l'éducation des filles. C'est là que le dessein de partir outreAtlantique va surgir progressivement. Religieuse cloîtrée, elle pense d'abord à prier pour les Amérindiens de Nouvelle-France dont elle reçoit les nouvelles sous la forme des Relations ${ }^{6}$ des jésuites. Peu à peu, elle réalise qu'elle devra se déplacer elle-même. Dans un premier temps, elle demeure discrète sur son projet : c'est un non-sens socioculturel qui ne peut lui attirer que des oppositions puisqu'elle est femme, cloitrée, d'origine modeste, provinciale, roturière. Mais le désir se fait pressant. Elle se décide donc à parler 
et convainc quelques personnes-clé, réussissant à contourner les obstacles majeurs qui ne manquent pas de se présenter; à commencer par les hésitations de sa propre supérieure et de ses principaux conseillers.

7 Après avoir mobilisé les ressources de ses réseaux sociaux - en activant des liens qui se trouvent porteurs de mouvements sociopolitiques innovateurs où s'inscrit son projet, tels ceux qui ont conduit à la création de la Compagnie des Cent-Associés pour le développement de la Nouvelle-France -, elle finit par s'embarquer le 4 mai 1639, avec Madeleine de La Peltrie, une bâilleuse de fonds libérale et non-conformiste qui, pour les circonstances, avait simulé un mariage avec Jean de Bernières, bien connu dans les milieux dévots ${ }^{7}$.

Marie déploie dans la petite colonie une activité intense, partagée entre l'éducation des jeunes filles amérindiennes et françaises, l'assistance aux Amérindiens, la fondation de la communauté des ursulines de Québec et un rôle de conseillère de plus en plus grand auprès des habitants de Québec et de la Nouvelle-France, à commencer par des jésuites et des administrateurs 8 . À cinquante-et-un ans, elle monte sur les échafaudages pour surveiller la reconstruction de son monastère, élevé dès 1645 mais détruit par le feu. Elle doit souvent s'opposer à des personnalités, parfois très proches, dont elle ne partage pas le point de vue et qui ne saisissent guère sa vision sociopolitique. Plusieurs fois alitée, elle écrit catéchismes, grammaires et dictionnaires en algonquin, montagnais, ouendat et iroquois. Elle meurt le 3 avril 1672.

La richesse d'une telle trajectoire sociale exigeait d'être partagée en phases temporelles propres à l'analyse. Nous en avons distingué sept: 1) enfance 1599-1617, 2) vie professionnelle 1617-1631, 3) religieuse cloitrée 1631-1635, 4) religieuse cloitrée... en apparence 1635-1639, 5) construction d'un monastère-école à Québec 1639-1650, 6) reconstruction et développements 1650-1663, 7) fin de la période pionnière, travaux de linguistique et vieillesse (1663-1672). La phase 4, la plus «bifurquante » a été choisie. Le choix de cette phase aura d'ailleurs (on y reviendra) une influence sur la qualité des données.

\section{2 - Identification des ressources mobilisées comme indicateur de réseau}

10 Dans cette phase spectaculaire, balisée temporellement par l'annonce du «dessein » (lettre de Tours du 20 mars 1635) et l'imminence du départ effectif (lettre de Dieppe du 18 avril 1639), nous avons voulu analyser les processus par lesquels Marie Guyart mobilise trois types de ressources indispensables: des informations et des autorisations (a), des biens (marchandises, services ou capitaux) (m), des ressources humaines $(\mathrm{p})^{9}$.

11 Sur la base des travaux de Luc Racine, inspirés de ceux de Marcel Mauss ${ }^{10}$, une méthode anthropologique s'est imposée à nous pour identifier un réseau propre à une analyse. Cette méthode est ancrée sur le corpus constitué par la Correspondance ${ }^{11}$ de Marie de l'Incarnation entre les deux dates concernées. Nous y avons repéré, une par une, les transactions (en termes anthropologiques, on dirait des échanges élémentaires) qui ont eu cours dans le cadre de l'opération de mobilisation de ressources menée par Marie Guyart pour réaliser le projet de fonder un monastère-école à Québec.

12 Ce travail de pré-analyse a consisté à noter, au fur et à mesure de la Correspondance, entre les deux dates jalonnant la phase 4 de sa vie, tout ce que Marie demande ou ce dont 
elle accuse réception, prouvant ainsi qu'elle l'a reçu ou que c'est accordé. Au fil des lettres, a été ainsi repérée l'arrivée des différentes ressources nécessaires à la mise en œuvre du « dessein » quelles qu'elles soient : avis (informations ou autorisations), biens (marchandises, services ou capitaux), personnes. On a noté chaque fois où Marie obtient quelque chose. On n'a considéré que les transactions qui entrent en jeu pour la NouvelleFrance. Tout ce qui n'a pas de lien direct avec l'action étudiée a été éliminé. On a distingué les ressources positives, relevé les obstacles et noté le nom de toutes les personnes participant aux transactions ${ }^{12}$.

Marie, agissant très prudemment de personne à personne, croise les groupes et les institutions et finit par rassembler ce qu'il lui faut pour son aventure, toutes ressources confondues. Ainsi, les caractéristiques de sa réussite pratique peuvent-elles être décrites en termes de nouvelles jonctions créatrices, selon ce que suggère Nohria : « elements are combined, taken apart, and recombined in a continuous process of organization formation and dissolution $»^{13}$. Ces éléments à combiner comprennent :

14 - un acteur qui a un projet innovateur (ici, Marie Guyart),

15 - de bonnes sources d'informations préliminaires (J.-A. Poncet),

16 - un bailleur de fonds principal (Madeleine de La Peltrie),

17 - d'autres bailleurs de fonds (Sillery),

18 - un gestionnaire (Jean de Bernières),

19 - des candidats à recruter pour l'équipe de départ (deux autres ursulines, encore Madeleine de La Peltrie, Charlotte Barré),

20 - des fournisseurs de ressources professionnelles ou autres services (Raymond, Françoise et ses ursulines, J. Dinet, G. de La Haye, de Meulles,...),

21 - une confidente proche (Ursule),

22 - des « supporters » extérieurs (Garnier et Chastellain).

23 Sans oublier l'obtention préalable d'autorisations essentielles :

24 - garantie spirituelle de Raymond,

25 - ordre de la supérieure, Françoise,

26 - caution morale des jésuites influents auprès des Cent-Associés,

27 - nomination par les Cent-Associés - ordre de mission de l'archevêque de Tours.

28 L'ensemble des ressources concernées (informations ou autorisations, argent, personnes) n'arrive pas tout à la fois. Elles se rassemblent, les unes après les autres, à la suite d'un développement aventureux, ponctué de péripéties où rien n'est gagné d'avance, mais qui permet de constituer un ensemble bien délimité où l'on peut repérer le nom des personnes qui ont participé à la mobilisation. Un tableau des graphes représentant les échanges élémentaires - d'informations, de ressources humaines et de biens - a été converti en une matrice récapitulative des pôles du réseau ${ }^{14}$ et en une représentation visuelle du réseau de Marie Guyart le jour de la décision politique de son départ (figure 1).

Une fois le réseau balisé avec un outil anthropologique, restait à savoir comment il avait « joué ». Comment l'acteur Marie Guyart gère-t-elle les réseaux où elle est impliquée en fonction de ses propres desseins? Nous nous inscrivons dans une perspective transactionnelle, plus active sur les réseaux et leur processus que la vision théorique « néostructurale »; et plus économique, voire utilitaire, que la vision anthropologique qui 
a permis de mettre en évidence un réseau d'échanges élémentaires. Nous allons regarder l'évolution de ce réseau comme une série de transactions entre joueurs unis par un projet parfois commun, selon le rythme de la partie; donc essayer d'identifier des stratégies de construction de réseau, sachant, bien entendu, que Marie n'aurait pu réussir si son entourage avait repéré chez elle la moindre trace d'ambition personnelle déplacée - ce qu'elle prévient en multipliant les expressions modestes invoquant sa condition « misérable » et la « bassesse de son sexe ».

Fig. 1 - Réseau de Marie activé le 11 janvier 1639

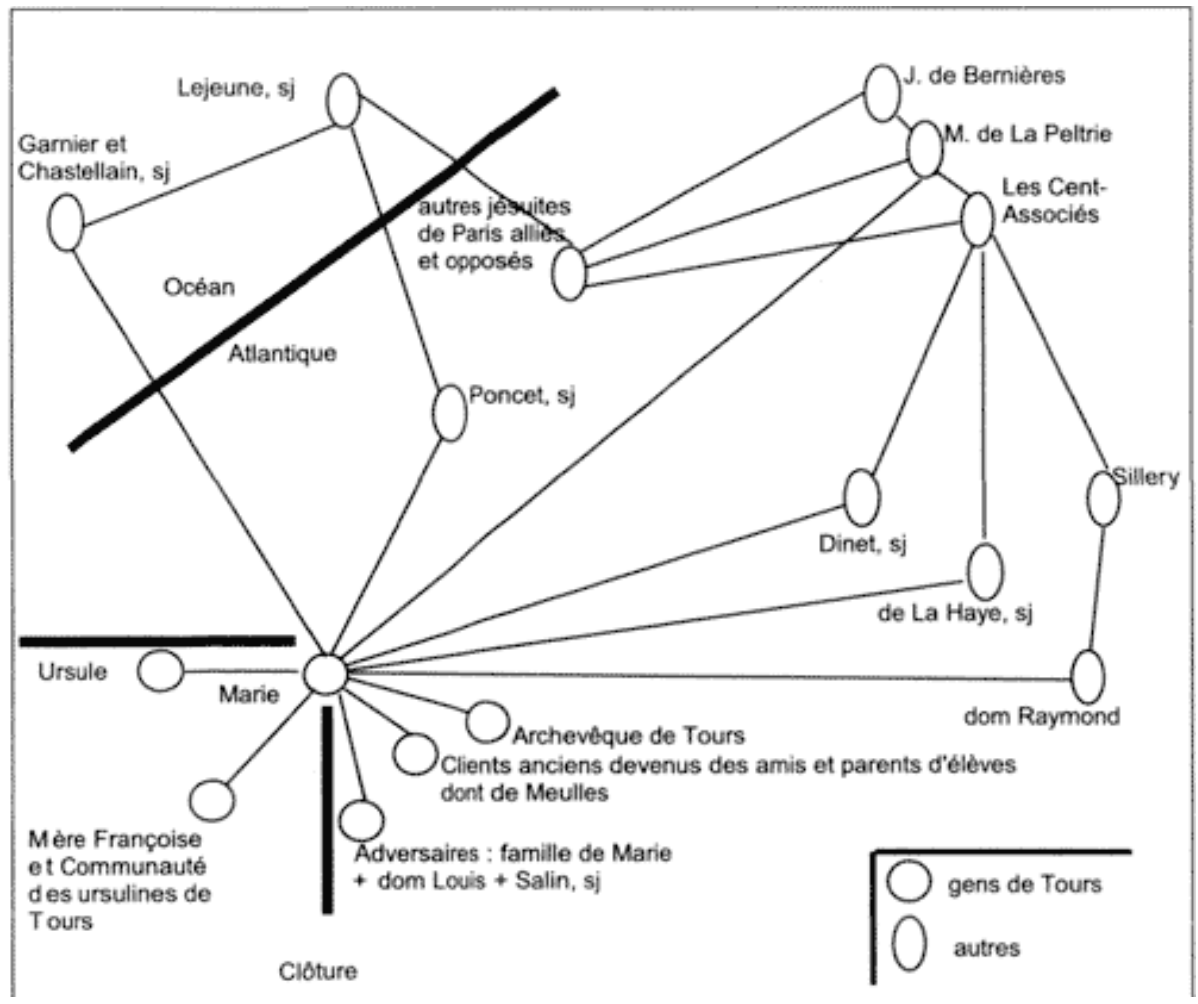

\section{Source et qualité des données}

Notre travail aurait pu souffrir, en principe, du fait qu'il se fonde principalement sur un compte-rendu de la situation provenant de l'acteur principal, et donc potentiellement biaisé $^{15}$. Mais ce n'est pas une reconstruction embellie a posteriori. Il s'agit d'un exposé au confident, dom Raymond ${ }^{16}$, où se précisent sans ambages les aides requises au fur et à mesure des événements. Par ailleurs, les informations recueillies à partir du texte de Marie pendant la période concernée ont été complétées ou précisées par les notes de l'éditeur, les lettres ultérieures complétant le corpus initial, laVie de Marie par dom Claude, son fils, ou d'autres documents historiques ${ }^{17}$. C'est dans ces sources complémentaires que nous avons aussi découvert quelques-unes des données manquantes et cherché à savoir où et comment se situaient dans la société les personnes et les événements repérés. Opération nécessaire en raison du style euphémique des lettres de Marie, femme discrète, qui sait ou pressent qu'il n'y a pas homogénéité d'opinion au sujet de son dessein, même à l'intérieur des groupes qu'elle connaît. Ainsi on a pu découvrir les noms de certaines personnes, notamment celles qui se sont opposées à son projet - dom Louis, Salin, sa famille. L'existence de ces personnes - qu'elle ne citera, 
lorsqu'elle décide d'en parler, que quinze ans plus tard dans son autobiographie (JII) et dont son fils et ses autres éditeurs éclairciront certains points après sa mort - souligne la difficulté de sa démarche ; les nommer en respecte la cohérence.

Il faut préciser - c'est bien connu - qu'il n'est pas possible de résoudre totalement la question des données manquantes dans une telle correspondance historique. Toutefois, les abondantes notes de l'édition critique permettent des recoupements qui valident les événements essentiels, surtout en ce qui concerne la partie française de cette correspondance, notamment la phase concernée dont les événements sont confirmés à la fois par l'autobiographie de 1654 et par des lettres postérieures. Par ailleurs, les lettres utilisées, très rapprochées dans le temps, comportent une certaine redondance dans l'exposé des démarches.

Une autre question méthodologique est celle de la perception que Marie pourrait avoir des relations entre autrui. Il s'agirait de données fragiles si elles étaient contemporaines à l'action ${ }^{18}$. Mais on a affaire à des événements du passé. La justesse de la perception est prouvée par ce qui doit survenir plus tard. De plus, comme dans le cas des données manquantes, on se fonde aussi sur le point de vue de l'éditeur et d'autres sources historiques.

Une troisième question concerne les raisons pour lesquelles il a été décidé d'envisager globalement, et non pas successivement, les trois types de ressources. Premièrement, cela aurait empêché de percevoir le jeu temporel du réseau qui s'est développé de façon multiplexe sur plusieurs terrains. Si l'on s'était éloigné de la chronologie, on aurait perdu le " fil rouge ", le caractère en spirale de la démarche, par bonds successifs ; l'obtention d'un certain type de ressource (une permission provisoire) permet d'aller chercher d'autres ressources (des services, puis des biens) qui débloquent l'accès aux autorisations définitives. Deuxièmement, nous avons voulu mettre l'accent, non pas sur les ressources, mais sur des processus de réseau, sur les caractéristiques d'une mobilisation réticulaire. Il fallait donc travailler par type de processus réticulaire plutôt que par ressource. Nous nous situons dans la foulée des observateurs de stratégie de réseau qui comporte deux aspects selon Herminia Ibarra (1992, pp. 165-168) : constituer et développer son réseau personnel et le mobiliser de façon sélective dans un but particulier.

Nous voulons pour notre part cerner ces processus au moyen d'un ensemble de propositions analytiques. Les premières concernent la structure et identifient la position de pivot qu'occupe Marie Guyart, au confluent de plusieurs réseaux ; d'autres envisagent la conjoncture et analysent sa compétence réticulaire, sa capacité de comprendre les réseaux et d'en utiliser les virtualités ; la troisième série de propositions est de l'ordre de la stratégie politique et met en relief certaines caractéristiques de la gestion du déroulement de son action réticulaire.

\section{3 - Une position-pivot dans la structure du réseau}

Les attributs sociologiques de Marie Guyart - femme, religieuse cloîtrée, roturière, provinciale éloignée des ports de mer, sans fortune - ne constituent pas a priori une recette gagnante pour mener à bien une expédition outre-mer onéreuse et politiquement risquée $^{19}$. Une bonne partie de sa force stratégique vient de ce qu'elle occupe une position-pivot: elle est une "étoile sociométrique " à la jointure de réseaux sociaux différents et antérieurs à l'action visée, qui renforcent son autonomie personnelle. 


\section{Une « étoile » sociométrique}

Les spécialistes des réseaux désignent ainsi une personne dont le point de représentation graphique est entouré de nombreuses arêtes ; on peut aussi la nommer " relais multiple » (Degenne et Forsé, 1994, p. 197). Cette "étoile » peut se situer au centre d'un réseau unique, mais elle peut aussi être à la frontière de plusieurs milieux qu'elle relie, dont elle reçoit des informations et à qui elle en donne. Elle peut jouer à ce propos le rôle d'amplificateur ou, au contraire, celui de filtre.

En termes simples, être une "étoile sociométrique » signifie qu'on a beaucoup d'amis et de relations, un «capital social $»^{20}$. Selon un des principaux analystes en ce domaine, Ronald Burt, « social capital is the final arbiter of competitive success » (1992, p. 58). En effet, beaucoup de personnes ont des qualités ou des savoir-faire équivalents. Il y avait parmi les jeunes ursulines de Tours beaucoup d'excellentes enseignantes volontaires pour partir en mission. «Toutes étaient frappées du désir de l'être... » (C, p. 447). Ce qui a fait la différence visiblement efficace en faveur de Marie, au bout du compte, n'a pas été forcément ce qu'elle savait, mais qui elle connaissait, dépendant sans doute de qui elle était, ce que nous aborderons plus loin. L'ensemble des personnes qu'elle connaît est vaste, corollaire du fait qu'elle est une femme aux multiples facettes, en partie illustrées par le statut varié des personnes qui l'ont aidée et dont le nom figure dans les schémas de ce texte - clercs, juristes, chapeliers, artisans, membres de la cour ou de la Compagnie des Cent-Associés - très différents les uns des autres. Nous allons en entrevoir un peu plus la variété et l'envergure en examinant la diversité de ses réseaux pendant la période antérieure à sa mobilisation de ressources pour partir en Nouvelle-France (figure 2).

Fig. 2 - Réseau de Marie pendant la phase antérieure à celle de la mobilisation de ressources pour partir en Nouvelle-France : 25/01/1631 - 20/03/1635

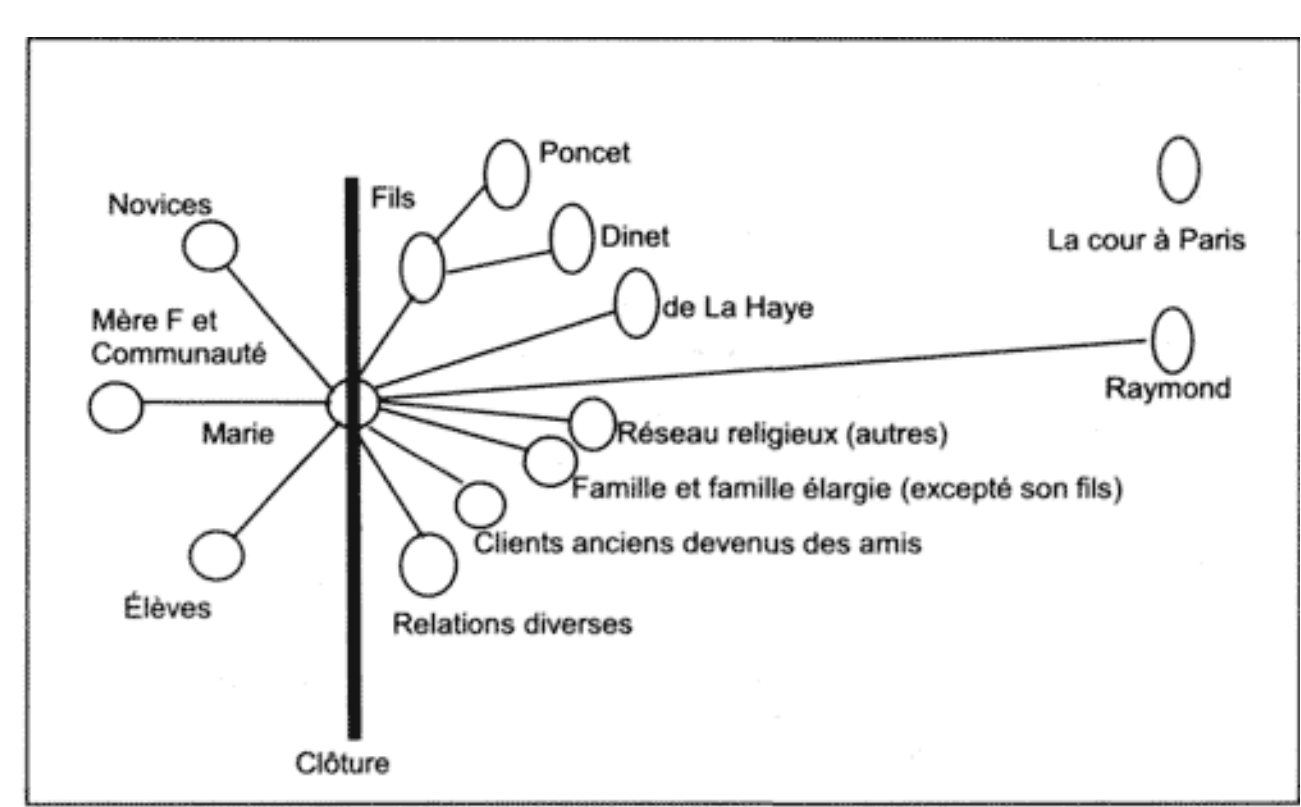




\section{À la jointure de réseaux sociaux différents}

38 À Tours, le réseau intérieur au cloître est constitué de trois catégories de femmes ; 1 - la communauté des religieuses, c'est-à-dire des collègues avec qui elle observe une règle précise ; 2 - les novices, qui ne sont pas encore religieuses et constituent l'avenir de la communauté ; 3 - les élèves, à former pour la vie civile ou pour la vie religieuse. Il y a peu de communication entre ces trois catégories. Marie est une des rares religieuses partie prenante des trois sous-réseaux à la fois, comme le prouve son curriculum vitae au monastère (M.-B. Rio, 1989, p. 39) (figure 3).

Fig. 3 - La vie de Marie Guyart de l'Incarnation chez les Ursulines de Tours.

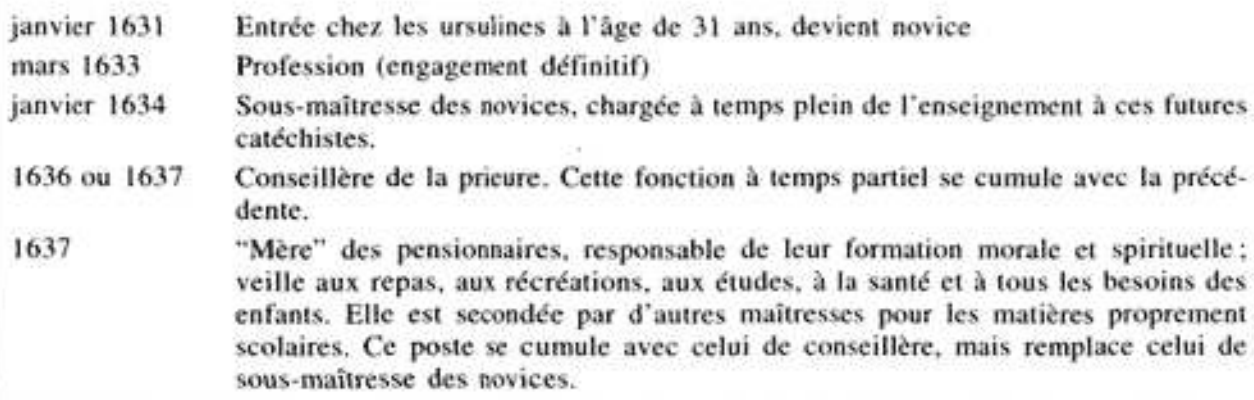
veille aux repas, aux récréations, aux études, à la santé et à tous les besoins des enfants. Elle est secondée par d'autres maîtresses pour les matières proprement scolaires. Ce poste se cumule avec celui de conseillère, mais remplace celui de sous-maîtresse des novices.

Ainsi en janvier 1639, Marie n'est plus en bas de l'échelle à l'intérieur du cloître. Elle est sur une pente ascendante et proche du sommet. Et elle a entretenu des relations privilégiées à la fois avec les religieuses, les élèves et les novices, qui forment trois réseaux assez distincts. On pourrait dire, en employant le langage de Burt (1995), que le réseau de Marie, dont la discrétion est bien connue (C, p. 1023), est riche en «trous structuraux $»^{21}$; elle est donc semblable aux directeurs les «mieux informés (qui) contrôlent des opportunités... (et) sont en position d'analyser l'organisation sociale pour définir des politiques; ils savent qui rassembler pour mettre en œuvre ces dernières... » (p. 607) 22 .

Vue de l'extérieur de la clôture, Marie a beaucoup moins d'importance : elle est - toujours - femme, religieuse cloîtrée, roturière, provinciale éloignée des centres de décision de Paris, sans fortune. Mais elle est entrée en "religion" sur le tard après une vie professionnelle active et ses réseaux sont demeurés différenciés et originaux, surtout pour son époque. Son réseau familial est double : celui de ses frères et sœurs et de leurs conjoints, dans l'ensemble opposés à ses projets, et celui de son fils, lui aussi opposé au projet, mais proche de ses professeurs jésuites et agent de liaison en quelque sorte malgré lui. Cette famille est opposée à un dessein qui n'a aucune pertinence raisonnable pour un Français ordinaire de cette époque. Le climat du Canada et la traversée de l'Atlantique font peur; ajuste titre. De plus, on confond la vallée du Saint-Laurent avec les Antilles où l'on envoie femmes faciles et détenues indésirables sur le sol métropolitain (C, p. 457). De là à montrer du doigt les familles des femmes parties outre-mer, il n'y a qu'un pas, que certains peuvent franchir. Aux réseaux familiaux très proches s'ajoutent les amis d'enfance et les cousins éloignés: Marie-Gillette Roland, l'amie d'enfance fondatrice des Visitandines de Tours, les cousines Chaussay, Carmélites à Tours, et d'autres. Les unes et les autres aideront Marie à Québec, dans d'autres phases de sa vie. 
41 Au réseau familial s'ajoute le réseau des clients et partenaires de l'entreprise de son beaufrère, qui a probablement joué même si la correspondance n'en porte pas directement trace. Nul n'a pu oublier son rôle de conciliation parmi les employés ou avec les clients ni sa plaidoirie réussie au tribunal de la ville en faveur d'un pauvre homme accusé injustement de meurtre et à qui elle a évité la potence.

42 À cela s'ajoute le réseau des parents des religieuses, des parents des novices ou des parents d'élèves que la charge de "mère des pensionnaires » ne peut que développer et renforcer. Sans oublier le réseau clérical partagé entre archevêché et clergé diocésain, cisterciens, jésuites, et autres ordres. Déjà, à Tours, ces réseaux se renforcent. Aux réseaux féminins, elle ajoute des réseaux masculins avec qui elle a l'habitude de discuter de façon autonome, avec prudence.

En dehors de Tours, les réseaux tourangeaux donnent accès à des réseaux nationaux et/ ou parisiens tout à fait spécifiques, en raison de l'histoire de la province. Comme ailleurs, les ordres religieux, Ursulines, Jésuites, Cisterciens, ont leurs propres antennes à Paris et dans toute la France. Mais, de plus, les gens de Tours sont spécialement bien placés à Paris. Cela remonte à l'époque où Louis XI avait fait de cette «bonne ville» la capitale de la France (fin du XV siècle). Depuis, les rois de France et les "grands", souvent en résidence au bord de la Loire et de ses affluents, ont pris l'habitude de recruter en Touraine une bonne partie de leur personnel (B. Chevalier, 1989, p. 9). Les fournisseurs suivent le mouvement. Les uns comme les autres peuvent assumer de grandes ou très grandes responsabilités, qui dépassent largement ce qu'on imagine à notre époque. N'oublions pas les relations internationales de Marie qui a pu accéder pendant sa mobilisation de ressources, par le père Poncet, à des liaisons directes avec le père Lejeune à Québec et avec des pères "aux Hurons » (Garnier et Chastellain, C, pp. 64-65). Enfin, après être passée à Paris, Marie entame des relations avec des membres des Cent-Associés et de la Cour, ce qui est tout à fait insolite pour une femme de son état.

Tandis que la plupart des gens développent des réseaux de relations avec des personnes semblables à eux, Marie, elle, bien que dans une position hiérarchique faible en dehors de son cloître, est reliée à des sphères sociales tout à fait différentes les unes des autres. Elle, plutôt en bas de l'échelle sociale de son temps, possède ce qu'on pourrait nommer simplement aujourd'hui un « capital social » différencié.

\section{Des réseaux initiaux antérieurs à l'action visée}

45 Marie n'aurait pas joui de la même crédibilité si elle avait pris contact avec les personnes qui lui ont permis de mener à bien son projet dans l'unique but de le réaliser. Au départ, ses liens étaient antérieurs et fondés sur d'autres bases. Dans tous ces réseaux, Marie est engagée dans des relations d'échange et de réciprocité. Les commentaires que son fils ajoute à ses récits de vie, tout comme les commentaires de ses proches après sa mort ( $\mathrm{C}$, p.1022), prouvent qu'elle a été une personne appréciée, qui a fourni de nombreux services, à un homme condamné injustement, aux employés de son ex-beau-frère, aux clients (JI, pp. 172-173), aux indigents ou aux malades (JI, p. 186). Ce n'est pas seulement pour lui être agréable et parce qu'elle est aimable qu'on l'aidera, mais aussi parce que bien des personnes sont prêtes à lui « rendre » ces services, matériels ou symboliques qui s'inscrivent dans toute l'histoire des relations antérieures. On peut y voir l'illustration du concept récent d'embeddedness ${ }^{23}$ en tant qu'il s'applique à l'efficacité dans les entreprises: "personal relationship... is determined largely by an history of 
interactions » (Granovetter, 1992, p. 35). Il est, par exemple, difficile d'imaginer que le parent d'élève Pierre de Meulles, proche du roi, aurait spontanément prêté sa maison à Paris s'il n'avait pas (bien) connu la qualité des services de Marie et de ses compagnes au point d'en être reconnaissant.

Fig. 4 - Relations de Marie avec les jésuites Dinet, de La Haye et Poncet en 1634.

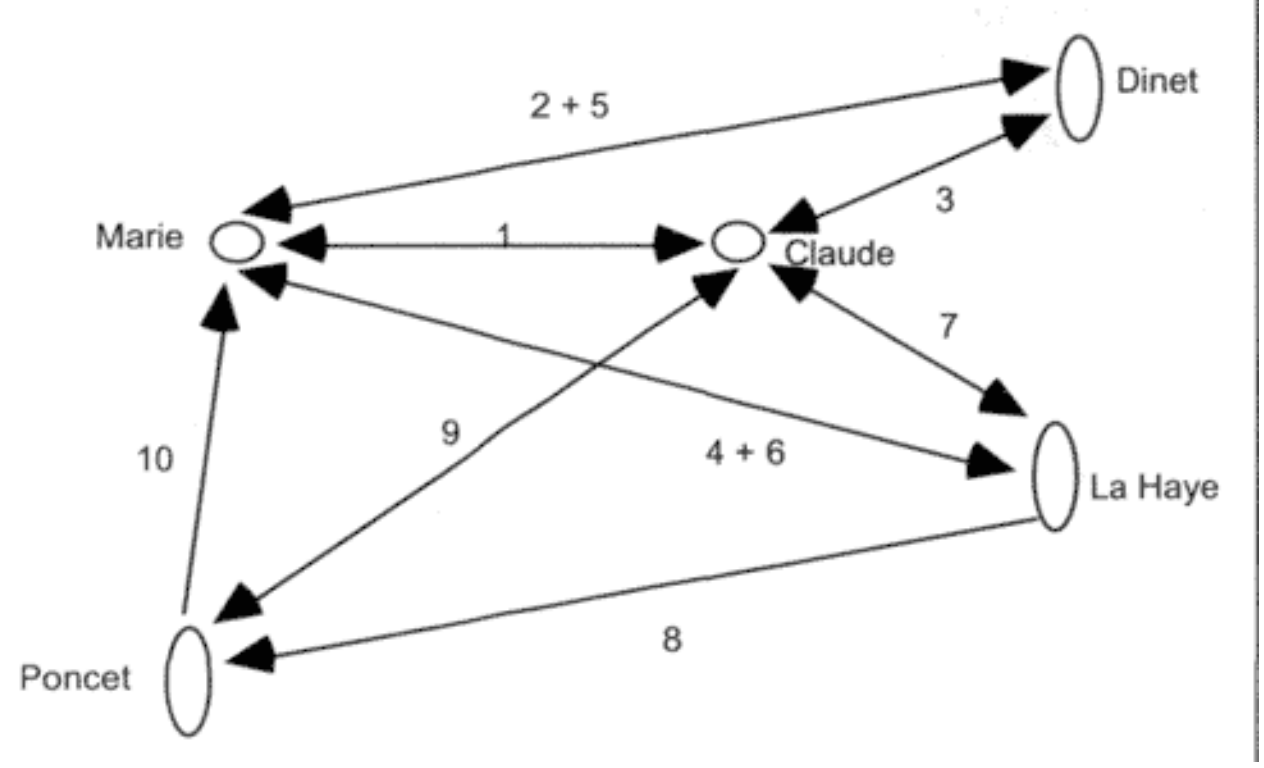

Le rôle muet de Claude, fils de Marie, est intéressant à observer, notamment dans les relations de sa mère, antérieures au dessein. Il n'est guère cité dans les lettres que nous envisageons. Mais Marie entretient des liens réciproques forts avec son fils, attestés en plusieurs endroits et que nous représentons dans la figure 4 par la flèche 1 à double orientation. C'est par l'intermédiaire de Claude que Marie a connu au moins un des Jésuites qui l'aideront beaucoup, son principal informateur, le père Poncet. Auparavant, Claude avait été l'occasion d'approfondir les relations réciproques avec les pères Dinet et de La Haye. Dinet, que Marie connaissait déjà en 1631, au point d'avoir eu plusieurs interactions réciproques (flèche 2 doublement orientée) se charge de conduire Claude (flèche 3) au collège de Rennes dont il est recteur à l'époque où Marie entre au couvent, en 1631 (JII, p. 185). Parallèlement Marie fait la connaissance de Georges de La Haye (flèche 4). Dinet revoit Marie à Tours au début de 1635 où il est alors confesseur des Ursulines. Marie peut donc discrétement exposer son projet (flèche 5). Elle a déjà éprouvé l'ouverture de Dinet. Elle l'estime. Vers 1633, c'est de La Haye qui est de passage à Tours et la rencontre (flèche 6). Il ne se contente pas d'encourager une lettre à Lejeune à Québec, il prend soin de faire avancer Claude (flèche 7) «en ses études qu'il avait commencées à Rennes " (JII, p. 184) et le met entre les mains de Poncet (flèche 8) à Orléans qui devient le principal professeur de Claude (flèche 9) en 1633. Se souvenant de Marie, une parent d'élève, c'est lui (flèche 10) qui a l'initiative de lui adresser en 1635 la Relation de 1634 (JII, pp. 205, 209 - C, p. 27).

\section{Une autonomie structurelle}

La diversité des réseaux de Marie Guyart assoit son autonomie structurelle au-delà de son talent. Elle peut d'autant mieux convaincre Raymond - réseau cistercien - qu'elle lui 
signale qu'elle a prévenu Dinet - réseau jésuite ; ou convaincre Françoise, sa supérieure, si Raymond et Dinet, conseillers influents, sont au courant. On retrouve ici une caractéristique des réseaux signalée par le pionnier de ces analyses, Georg Simmel, et par un chercheur contemporain, Emmanuel Lazega :

«La multiplication des cercles sociaux, en multipliant pour les acteurs les opportunités d'arbitrage entre différents ressorts d'action, développe leur autonomie et leur capacité d'initiative et d'innovation. » (Simmel, 1917)

«...l'autonomie et la centralité des acteurs... peuvent être considérées comme un indicateur de leur capacité d'innover : un acteur autonome a davantage de chances de pouvoir créer des coalitions capables de promouvoir ce changement. » (Lazega, 1994, p. 310).

Grâce à cette autonomie, elle peut pousser ses ramifications très loin dans les milieux des pouvoirs économiques et politiques qui l'intéressent. En cas de défaillance d'une ou plusieurs connexions, par exemple Dinet, Raymond et Françoise - ce qui arrive de mai à octobre 1635 - la redondance de certaines relations - par la Haye, en mars 1636 l'autorise à rejoindre Lejeune sans passer par les connexions caduques et lui permet de demeurer branchée malgré un environnement changeant. M. Forsé $(1991,1994)$ constate précisément, dans sa typologie des étoiles sociométriques, que l'étoile, si elle est fortement dépendante, est aussi fortement motrice, c'est-à-dire munie d'une certaine marge d'autonomie. Analysons donc maintenant l'activité de cette femme d'entreprise.

\section{4 - La lecture de la conjoncture : compétence réticulaire de Marie Guyart}

Marie Guyart est une femme d'entreprise, qui repère dans chaque personne rencontrée, au-delà du rôle qui motive le premier contact, les autres rôles potentiels de cette personne. Elle possède une compétence communicationnelle, guidée par une perception « sociologique » de la société, qui lui permet de convaincre et donc d'actualiser des rôles sociaux à venir. Elle sait également piloter des transactions réticulaires explicites.

\section{Une compétence communicationnelle}

Marie sait entrer en relation avec autrui. Elle pratique la chimie des communications, comme le dit Nohria (1992, p. 242). Par exemple, sachant que la jeune sœur Marie de Saint-Joseph a les qualités requises pour l'accompagner en Nouvelle-France, elle arrive vite à la convaincre de présenter sa candidature :

«À la première parole que je lui dis, toutes ses peines (réticences) furent dissipées »

(C, p. 448).

51 Le fait est que, capable d'écouter, de recevoir des informations et d'en exprimer, elle se comporte comme une personne dont on se souvient après lui avoir parlé. Sinon, elle n'aurait jamais été informée de la possibilité pour elle de partir « en Canada » auprès des « peuples les plus pauvres du monde » qu'elle n'imaginait rencontrer « qu'en esprit » (JII, pp. 198-203).

52 En effet, c'est parce qu'elle a impressionné un interlocuteur occasionnel, en l'occurrence un professeur de son fils, Joseph-Antoine Poncet, que ce dernier lui envoie en 1635 la Relation des jésuites de Québec qui demande des «maîtresses" pour les jeunes Amérindiennes. Non seulement Poncet adresse cette Relation, mais il propose à Marie de 
poser sa candidature. Rappelons que, dans le contexte de l'époque, ceci est une aventure absolument exceptionnelle, « en apparence contre toute la raison humaine » (C, p. 30), qui figure parmi les « choses extraordinaires » (C, p. 447). Le simple ${ }^{24}$ contact professeur/ parent d'élève a donc, dans ce cas, marqué le professeur au point que ce dernier l'invite, sans savoir si Marie est, oui ou non, intéressée à poser un acte aussi innovateur.

Si Marie est une personnalité remarquée - par certains - à l'extérieur de son monastère, elle l'est encore plus à l'intérieur où on lui a confié des responsabilités de contact et d'influence. On aime la rencontrer. Les novices lui redemandent des « instructions». Ses prestations plaisent. Il lui sera ordonné de les consigner par écrit ${ }^{25}$.

Elle exerce un charisme certain : pour elle, on sera prêt à réexaminer un point de vue, voire à changer d'avis, alors même qu'on n'était pas d'accord avec son projet. Ainsi, Raymond, son maitre spirituel, dont l'autorisation est absolument nécessaire pour entamer les démarches, commence par la rabrouer en lui reprochant son « imbécillité » ${ }^{26}$. Elle le persuade et il se transforme en personne-ressource des plus efficaces. De même, Françoise, la supérieure, qui soutient le projet, puis s'y oppose devant la levée de boucliers venant de clercs locaux, est finalement conquise et devient, elle aussi, une ressource hors-pair. Même les clercs locaux ennemis changeront d'avis juste avant le départ (JII, p. 220).

\section{Une perception « sociologique » de la société}

Au-delà de sa capacité de convaincre, Marie Guyart n'aurait pu arriver à ses fins si elle n'avait su percevoir, jusqu'à un certain point, les rapports sociaux de son temps dans leur structure établie et dans leurs potentialités mouvantes. Elle a conscience des rôles, elle sait comprendre l'organisation sociale et la place que chacun y occupe. La manière dont elle décrit le parcours de Madeleine de La Peltrie est comme une petite anthologie du genre.

« Elle fut à Caen en secret pour consulter Monsieur de Bernières, qui l'encouragea puissamment... Son cœur se sentant extraordinairement pressé d'exécuter son dessein, elle s'en alla à Paris pour en chercher les moyens, et Monsieur de Bernières l'y fut trouver pour l'aider en cette recherche... Ceux qui furent principalement consultés sur une affaire si extraordinaire furent le père Gondren et Monsieur Vincent... L'un et l'autre ayant jugé que cette vocation... était de Dieu ${ }^{27}$, Monsieur de Bernières ne pensa plus qu'à chercher le père qui faisait à Paris des affaires du Canada $^{28} \ldots$ On consulte les révérends pères Lalemant et La Haye ${ }^{29}$, et par leur conseil Monsieur le Commandeur de Sillery et Monsieur Fouquet ${ }^{30} \ldots$.. (qui écrivent des lettres de recommandation à l'archevêque de Tours) que l'on croyait devoir être inexorable pour un dessein si extraordinaire, (et qui) après l'avoir entendu parler et vu les lettres... fut comme ravi de la grâce que Dieu lui faisait de prendre deux de ses filles pour une si glorieuse entreprise... » (C, pp. 906-907-908)

On voit ainsi défiler, sous la plume de Marie Guyart, et dans le bon ordre, toutes les personnes essentielles qui jalonnent son parcours et, aussi, celui de Madeleine de La Peltrie: Jean de Bernières, support personnel et conseiller en gestion, les experts psychologiques, garant de son équilibre, les trois jésuites - La Haye, Lalemant et Poncet -, tenants des rôles de «portiers" pour les «affaires du Canada» et, enfin, les représentants des Cent-Associés, qui détiennent le pouvoir décisionnel.

L'ordre des personnes proches auxquelles Marie Guyart s'ouvre de son projet est un autre aspect de sa compréhension de l'organisation sociale; quatre sont dans la confidence, 
triées sur le volet : Raymond, le maître spirituel de sa jeunesse, sans doute son meilleur ami, et, sans qu'elle le sache forcément, un homme très proche des Cent-Associés; Françoise, sa supérieure; Ursule, l'alter ego maîtresse des novices de sa communauté, chez qui elle distingue - peut-être - déjà une future prieure ; et Dinet, qu'elle connaît bien - elle lui a confié son fils au collège des jésuites de Rennes en avril 1631 et il a été un confident important; il est aussi - le sait-elle ? - confesseur du roi Louis XIII ${ }^{31}$. Raymond et Françoise sont les plus importants au départ. Ce sont les premiers "portiers", incontournables, sans l'accord de qui elle ne peut rien réaliser. De plus, Françoise, en tant que supérieure, détient les clés de l'affectation des «obédiences » des religieuses qui pourraient la seconder dans l'aventure.

Les premières lettres parlant du " dessein » s'adressent à Raymond. Le ton utilisé dans ces lettres - notamment les formules de politesse - prouve combien elle évalue et respecte la situation de chaque personne dans cette société d'ordres hautement hiérarchisée où elle se situe en bas.

«... je m'imagine que vous m'allez blâmer de ce qu'étant si misérable, j'ose aspirer à une vie si sublime» (C, p. 25) «Et je ne m'étonne pas si vous êtes surpris et dans l'étonnement de me voir aspirer à une chose qui semble inaccessible, et encore de voir que c'est moi qui y aspire. » (C, p. 26)

Il est bien évident qu'elle a enregistré l'information nécessaire sur les rôles dans la communauté et parmi les clercs qu'elle fréquente, comme elle a appris à le faire parmi les clients en tant que gérante de l'entreprise de transport de son beau-frère. Mais elle va bien au-delà.

\section{La transposition des caractéristiques personnelles et réticulaires dans les rôles sociaux à venir}

En d'autres mots, Marie comprend la dynamique des personnes au-delà de l'apparence de l'instant. Elle anticipe et planifie. Pourvue d'imagination "sociologique $»^{32}$ et de la capacité de repérer les mouvements et les émergences, elle sait que derrière chaque personne rencontrée dans un rôle, d'autres réalités ou potentialités se profilent. Ainsi, chez Raymond, partie prenante des préjugés mâles de sa société33, Marie sait que se cache non seulement un homme sage qui n'osera pas la condamner entièrement, mais aussi quelqu'un qui se trouve à l'intersection de groupes sociaux différents possédant l'information et les ressources dont elle doit disposer pour son projet. Elle ignore sans doute encore, au moment de sa première lettre de demande d'aide, qui il peut contacter précisément et ce qu'il peut procurer, mais elle est consciente qu'il peut beaucoup.

61 Plus tard, on l'a vu lors du choix de sa compagne, elle opte pour la jeune Marie-Joseph. Ce n'est pas évident. Il s'agit de ne pas se tromper sur la personne qui devra la seconder pour la fondation d'une œuvre inédite. Or Marie-Joseph (née de Savonnières de la Troche de Saint-Germain) n'a pas l'apparence des qualités requises. Elle avait été atteinte d' "asthme et fluxion sur le poumon» (C, p. 438) et demeure de santé fragile. Pourtant Marie Guyart perçoit chez la jeune femme, au-delà de l'aspect frêle et d'une appartenance à la vieille noblesse propice aux vanités, toutes les qualités d'entregent et de contact qui seront nécessaires dans un pays rude et avec les Amérindiens.

Par ailleurs, Marie aurait pu accepter l'argent de Madeleine de La Peltrie, mais ne pas opter pour une compagnie aussi originale. Au contraire, elle reconnaît chez sa bâilleuse de fonds la personne qui devra l'accompagner. Par contre, la suivante de Madeleine de La 
Peltrie s'est découragée avant de quitter Tours et Marie n'a pas insisté pour la convaincre; elle s'est plutôt empressée d'en chercher une autre, Charlotte Barré (JII, p. 240), chez qui, l'expérience le prouvera, elle a tout de suite décelé les qualités ad hoc.

\section{Le pilotage de transactions « réticulaires » explicites}

63 Le projet avançant, Marie doit aussi utiliser, pour parvenir à ses fins, des stratégies explicites :

«Ce n'est donc pas rechercher nos propres intérêts que de recourir à ceux que nous croyons que Dieu a mis en ce monde pour nous aider. « (C, p. 39)

Si l'opération de mobilisation des ressources a pu réussir en dépit des contraintes structurelles, conjoncturelles ou politiques, c'est que Marie s'est souvent posé une question, plus ou moins consciemment, à chaque rencontre depuis le début du projet: cette personne pourrait-elle procurer certaines ressources nécessaires? Et cela, malgré les contraintes envisagées? Pourrait-elle même diminuer ces contraintes? Marie, exfemme d'affaires, peut à l'occasion utiliser soit ses capacités de négociatrice, soit des actions indirectes, comme aux échecs.

Pour persuader Raymond, elle négocie sur leur terrain d'entente profonde, avec des arguments mystiques. La manière dont elle obtient la nomination de la jeune sœur Marie de Saint-Joseph pour l'accompagner est un exemple à la fois d'anticipation de rôle et d'action indirecte (C, pp. 446-449). Une commission de sélection avait été constituée de trois hommes et trois femmes: le supérieur ecclésiastique des ursulines, Françoise, prieure des ursulines, M. de La Peltrie, Bernières et Marie. Les candidates sont priées de donner leur nom à la prieure. Celui d'Ursule n'apparaît pas dans la liste : ses parents s'y sont formellement opposés. Personne ne semble avoir fait de démarche pour qu'ils changent d'avis. Par contre, malgré ses vingt-deux ans et sa santé toujours fragile, la jeune Marie de Savonnières s'est inscrite. Elle est devenue une jeune femme pleine d'entrain, indépendante, fière et d'une grande liberté d'esprit : exactement la personne qui convient aux yeux de Marie Guyart. Mais sa candidature est immédiatement rejetée par la prieure : trop jeune, trop frêle.

Marie est trop perspicace pour faire valoir son point de vue dès le départ. Après étude cas par cas, toutes les candidatures sont écartées par un membre ou l'autre de la commission. Alors notre diplomate entreprend un lobbying. D'abord, elle souligne les qualités de la jeune sœur à $\mathrm{M}$. de La Peltrie et à Bernières, et s'assure de leur appui. Puis elle va trouver Françoise "avec des insistances fortes et respectueuses». La prieure n'en dort pas pendant toute une nuit et finit par accorder son consentement.

Nous avons vu que Marie Guyart était, structurellement parlant, une personne-pivot, à la jointure de plusieurs milieux et réseaux sociaux. Nous venons de constater qu'elle était compétente en tant que femme d'entreprise sachant utiliser la conjoncture, capable de conduire un projet, de convaincre et de recruter pour ce dessein. On peut de plus, à travers son cas, formuler des propositions relatives à la stratégie politique des réseaux sociaux. 


\title{
5 - Quatre règles de stratégie politique réticulaire
}

\author{
L'utilisation de liens forts, puis de liens faibles
}

Depuis l'article de Granovetter (1973) ${ }^{34}$, les analystes des réseaux s'entendent pour qualifier les liens sociaux de forts ou faibles, selon l'intensité de ce qui unit deux personnes. Granovetter a montré que les liens forts tendent à relier en groupes étroitement connectés des gens similaires; l'information circule de façon redondante parmi eux, ce qui ne permet guère de pratiques innovatrices. Par contre, un lien faible constitue une passerelle permettant, si elle est opérante, d'échapper à un système social clos et de se procurer une information renouvelée. Granovetter (1973, p. 1361) propose quatre caractéristiques du lien fort: selon le temps, l'intensité émotionnelle, l'intimité (confiance mutuelle) et les services réciproques rendus.

De nombreux auteurs, depuis 1973, ont relevé des ambiguïtés dans cette définition en partie subjective, ce qui n'a empêché personne de continuer à utiliser cette catégorisation en essayant de la raffiner. Les travaux sur l'intensité évaluée selon différents critères (nomination réciproque ; temps ; ami, connaissance ou voisin) sont nombreux (C. Bidart, 1991, pp. 21-42 ; D. Krackhardt, 1992, pp. 216-239). Ils désignent cependant des objets sociologiques ambigus car ils renvoient à des critères difficiles à pondérer: l'amitié, le respect ou la confiance, le prestige ou la sympathie éprouvée dans des situations exceptionnelles, voire une utopie partagée, parfois mystique. Parmi les amis, Krackhardt (1992, p. 218) distingue les philos, qu'il définit par des liens forts impliquant trois conditions nécessaires et suffisantes: interactions réciproques, affection réciproque, durée significative.

La littérature sur les réseaux sociaux classe habituellement le confident comme un lien fort. Nous aurions pu développer ce point en soulignant dans la vie de Marie Guyart le rôle d'Ursule qui n'a jamais cessé de la soutenir, même quand tous les autres l'abandonnaient. Toutefois, nous allons retenir la distinction plus fine de philos et remplacer la notion d'affection réciproque par celle d'utopie réciproquement partagée ${ }^{35}$, doublée d'un niveau de confiance élevé. De tels liens forts se sont établis entre Marie Guyart et cinq personnes, soit Françoise, sa supérieure hiérarchique directe, sa compagne Ursule, Raymond, puis Dinet et de La Haye.

Bien sûr, nos données confirment la pertinence de l'analyse de Granovetter en ce qui concerne l'arrivée en avril 1635 de la première information sur la Nouvelle-France, par un lien faible, Poncet, simple professeur de son fils. Mais c'est plutôt au moyen de ses liens avec les trois philos extérieurs à son cloître que Marie a pu, par personnes interposées, gagner les instances qu'elle devait rejoindre.

Reprenons la figure 1, qui décrit le réseau activé en janvier 1639. On constate que Marie, en situation de faiblesse, a d'abord pris contact avec les philos extérieurs au cloître (Raymond, Dinet, La Haye) qui, eux, ont fait jouer d'autres liens en faveur de son projet. Religieuse cloîtrée, il lui était parfaitement impossible d'accéder à qui que ce soit sans passer, d'abord, par ces personnes avec qui elle entretenait des liens forts et qui se sont avérées des relais efficaces. Si bien que nous pouvons emprunter à Krackhardt sa conclusion : 
"A major ressource that is required to bring about such change is trust in the propaga-tors of that change. Change is the product of strong, affective, and timehonoured rela-tionship. Change is the product of philos » (1992, p. 238).

Ce qui n'empêche pas des relations faibles d'apporter des informations ou d'autres ressources nécessaires.

\section{L'utilisation de personnes-relais} ressources circulent d'une personne à une autre, et au moment où s'engage une transmission dans une chaîne de liens faibles, le message risque de s'atténuer et de se perdre. Lorsque Marie parle à ses trois philos hors clôture, ils peuvent soit jouer le rôle de filtre et garder l'information pour eux donc ne pas faire avancer le projet, soit jouer celui de relais amplificateur, ce que font Raymond, Dinet et La Haye.

Marie ne sait probablement pas, lorsqu'elle informe Raymond de son projet pour la première fois en 1635, qu'il connaît bien le richissime commandeur de Sillery, ou que le supérieur de Raymond, Lauson, est le frère de Lauson, propriétaire de l'île de Montréal, un des directeurs des Cent-Associés (V, p. 339 - G. Oury, 1973, p. 264). Mais elle sait que Raymond a des «connections » et qu'il peut l'aider, bien au-delà du conseil spirituel. Ce qu'il va faire. C'est, par exemple, par Sillery, un ami très discret de Raymond, que Marie obtient, non pas toutes les ressources nécessaires, mais la première, un terrain sur le Cap Diamant. Nul ne sait le rôle joué par l'antenne de Sillery aux Cent-Associés, ni par Jean de Lauson, mais il est probable qu'un avis favorable soit arrivé par cette piste.

Observons (figure 5) le processus d'acquisition d'une concession à Québec pour un monastère-école de filles. C'est le résultat positif d'une chaîne de relais activée dans le temps et dans l'espace.

Ce terrain à Québec, première ressource obtenue, est une conséquence d'une des toutes premières lettres de Marie dans la phase observée. Elle demande (a) alors à Raymond, son premier philo, comment procéder pour réaliser son dessein. Il l'encourage et en parle (b) à Sillery. Ce dernier, qui s'inscrit dans la ligne des mystiques « fous » de l'époque, est prêt à financer toute l'opération de Marie ; mais son argent est « détourné » pour fonder une « réduction » en un lieu qui portera son nom.

Toutefois il joue positivement son rôle de relais et l'idée du «séminaire » pour jeunes Amérindiennes fait son chemin, par l'intermédiaire (c) d'un ami de Sillery, Jean de Beauvais, qui rejoint (d) les Cent-Associés, pouvoir parisien sur la Nouvelle-France. Marie en est avisée (e) et (f) par un intermédiaire inconnu. La concession est accordée le 15 janvier 1637 pour des religieuses enseignantes et un « séminaire» de filles (C, p. 50 et C, p. 63). Cette concession passera aux ursulines de Québec en 1639. 
Fig. 5 - Représentation graphique de l'acquisition d'une ressource.

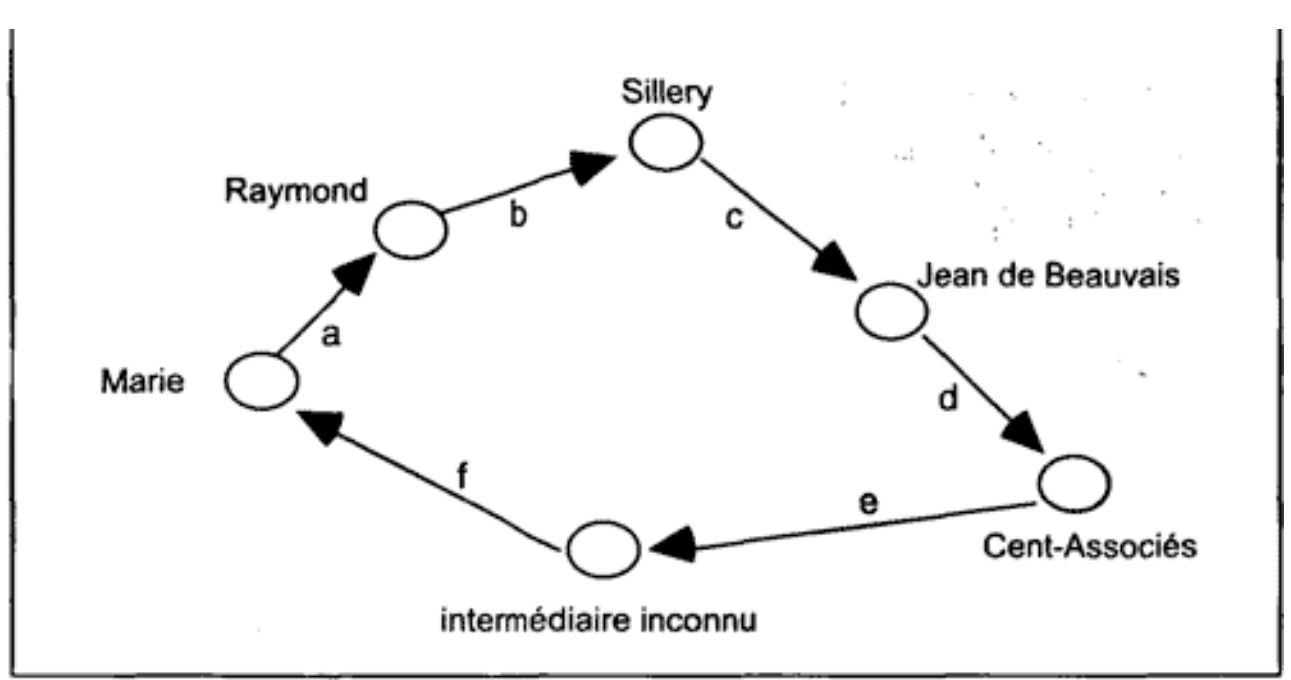

\section{Couplage et découplage : des réseaux étanches, à discrétion}

La notion de connexité d'un réseau peut être abordée par la question : comment peut-on rejoindre quelqu'un, en utilisant ses relations ? Dans les organisations plus hiérarchiques, on ne peut en principe rejoindre quelqu'un qu'en passant par le haut. Dans celles qui comprennent d'autres réseaux que ceux de la hiérarchie, y compris des philos, on communique plus facilement. Certains parlent de réseaux étanches ou intransitifs et de réseaux transparents ou transitifs. Par ailleurs, Granovetter (1973) a montré comment c'est sa «triade interdite »- si A et B se connaissent et que A et $C$ se connaissent, il est très difficile que $B$ et $C$ ne se connaissent pas. Or, dans les stratégies de réseaux, il est parfois nécessaire de ne pas amener $B$ et $C$ à se rencontrer, et plus généralement, de ne pas connecter des éléments du réseau qui pourraient être opposés sur d'autres points. C'est une importante stratégie de couplage/découplage.

Marie Guyart a dû, entre l'interdiction de sa supérieure en octobre 1635 et la nouvelle autorisation, vers janvier 1639, découpler complètement ses relations à l'intérieur du monastère concernant son travail du moment et ses relations extérieures concernant son projet. Elle sait passer de l'un à l'autre réseau par une mise en latence apparente du projet dans le réseau intérieur et une construction discrète de ce projet par les relais de ses philos. Elle acquiesce à l'impossibilité d'exécuter le dessein, mais «sans pourtant perdre la volonté de l'embrasser» (C, p. 56). En effet, trois mois plus tard, elle propose sa candidature à Le Jeune à Québec, concluant : « ... il n'y a rien en ce monde qui m'en puisse empêcher quand même je devrais être engloutie des ondes en chemin. » (C, p. 60)

Entre les deux moments, elle a contourné, sans le faire délibérément, on va le voir, l'interdiction de Françoise qui lui « dit dans l'affection qu'elle me porte que je ne vaudrais rien du tout en Canada... » (C, p. 51) et celle du confesseur local, Salin, à qui elle a parlé de ses désirs sans que la réponse ne se fasse attendre :

« Il me fit taire quasi dès le premier mot et me mortifia bien sec, se moquant de moi qui m'amusais, disait-il, à des fantaisies. » (JII, p. 214)

Elle saisit l'occasion au vol. Dès qu'on le lui interdit, Marie ne parle plus de son projet. Jusqu'au jour où elle reçoit la visite d'un jésuite, probablement au sujet de son fils Claude, 
par une voie tout à fait différente de la voie hiérarchique. Elle lui parle. Il répond qu'elle est obligée « en conscience » de communiquer toute son affaire à La Haye. Elle ne se fait pas prier et profite de sa lancée pour proposer ses services à Le Jeune, supérieur de la mission de Québec (JII et V, p. 347).

Autre exemple de couplage/découplage, l'approche des Cent-Associés via ses philos, Dinet et de La Haye, qui jouent le rôle de relais. Il y a, parmi ces Cent-Associés, des chapeliers aux visées économiques, des mystiques et des parisiens. Ces trois ensembles peuvent se recouper. La plupart des chapeliers et des mystiques sont parisiens et une fraction de chapeliers peut, dans la mentalité du temps, respecter une « sainteté » qui, par proximité, pourrait rejaillir sur eux. Mais on peut supposer que d'autres chapeliers ne sont guère mystiques, donc prêts à se rallier aux arguments contre Marie en faveur de religieuses parisiennes, apparemment plus fonctionnelles, la candidature de la tourangelle étant fort mal vue de certains parisiens, tel Binet, un adversaire déclaré de la mission de NouvelleFrance ${ }^{36}$; quitte à envoyer des ursulines, il connait mieux les parisiennes. Pour faire valoir la candidature de Marie Guyart, il est donc nécessaire de passer au-delà de l'étanchéité relative entre le réseau mystique et les réseaux économiques ou religieux, sans toutefois rendre le dessein mystique par trop transparent à des décideurs qui pourraient s'en inquiéter et le contrecarrer. En cette affaire, le relais est pris par La Haye et Dinet, bien placés pour connaître les qualités de femme d'affaires de Marie Guyart, qui seront utiles à Québec, et opérer une synergie de différences (JII, pp. 229-321) ${ }^{37}$. Cet exemple évoque des remarques contemporaines de R. Canter-Kohn :

«Le réseau m'intéresse aujourd'hui en tant que structure sociale de mise en synergie des différences, de liaisons entre termes indépendants, de rapports entre l'identification à un espace et le remplacement de celle-ci au profit des liens. Autrement dit, le réseau comme structure compréhensible alternativement à deux niveaux différents et selon des points de vue contradictoires. » (1993, p. 43).

C'est dans une « articulation/disjonction que nous pensons pouvoir lire les conjonctions et les conflits d'intérêt et les négociations entre logiques et pratiques différentes...» (1992, p. 132)

\section{Des stratégies diversifiées}

Forte d'un réseau varié dont elle connaît les virtualités, Marie (et ses philos) est en mesure de mettre en œuvre des stratégies plus raffinées pour tourner à son avantage une situation qui présente des risques considérables.

\section{Recherche ciblée d'une personne rare}

Marie cherche une personne assez « folle " pour subventionner à fonds perdus la création de son école pour jeunes Amérindiennes en Nouvelle-France et qui aurait toutes les qualifications requises pour faire face aux nécessités d'un projet particulièrement exigeant. On pourrait penser qu'elle et ses philos mènent alors une opération de stratégie extensive, parcourant en tout sens un vaste territoire pour découvrir la perle rare. Ce n'est pas ce qui se passe. La perle est si rare qu'on ne peut la rejoindre avec des moyens de communication de masse de l'époque (ce serait, par exemple, des annonces aux prêches), même en se limitant au milieu dévot. Il faut faire fonctionner une stratégie spécifique, large mais sélective, en visant des canaux précis de diffusion de l'information, par exemple, les lecteurs des Relations. Les philos sont bien placés, diffuseurs ou attentifs 
lecteurs de ces Relations et confidents de personnes riches et généreuses qui pourraient convenir. Justement, Raymond contacte Sillery, ce qui est un succès partiel - pour le terrain -, mais un échec pour la dotation. C'est Poncet, un lien faible, celui qui avait envoyé la Relation et l'« offre d'emploi » de la Relation de 1634 lue en 1635, qui met en relation avec Madeleine de La Peltrie; celle-ci se livrait, de son côté, à une démarche similaire et avait repéré l'annonce placée par Lejeune, de Québec, dans sa Relation de 1635 lue en 1636. C'est Lejeune qui a mené la stratégie efficace; et Marie en a profité par Poncet.

Fig. 6 - Tentatives pour découvrir un(e) bailleur de fonds.
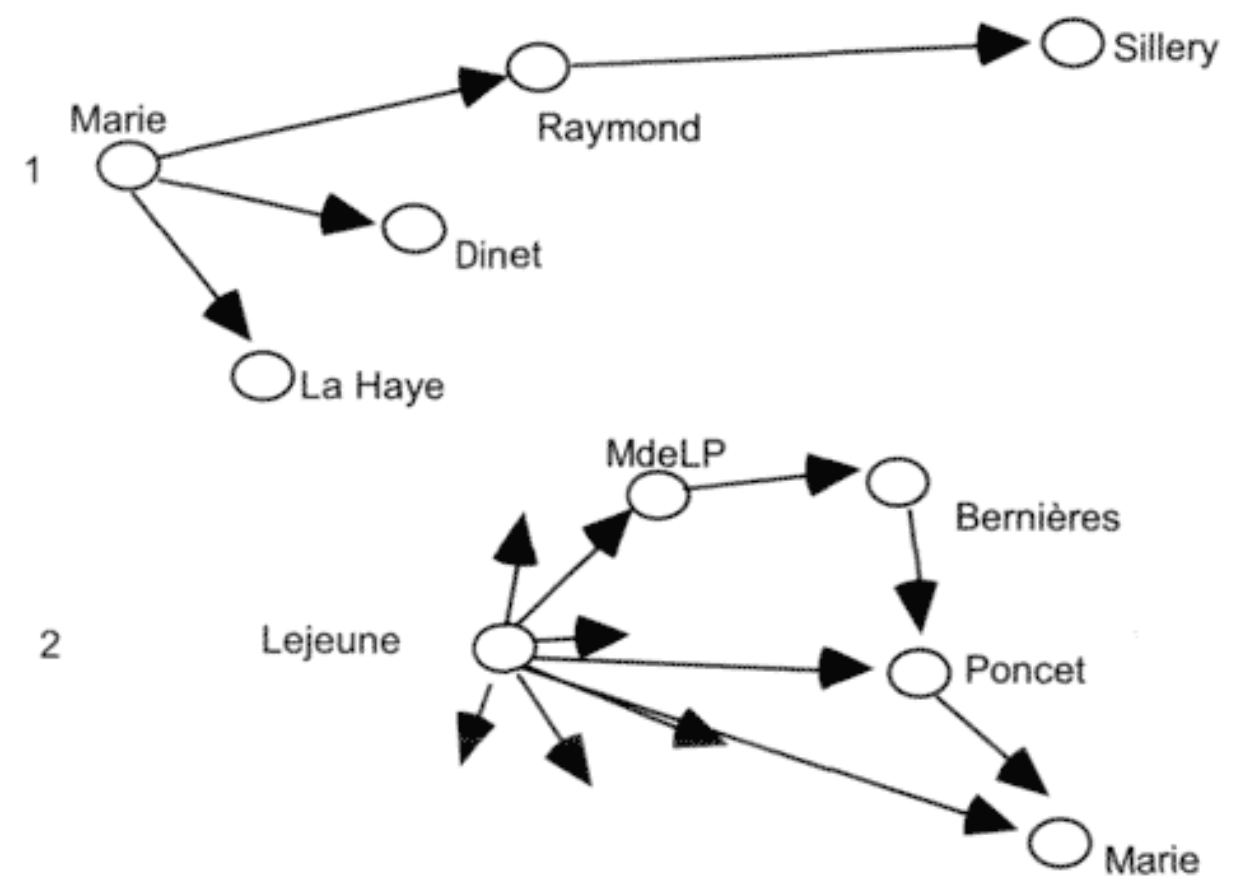

La « découverte » de Madeleine de La Peltrie est donc un processus spécifique, qui peut se résumer ainsi (figure 7) : Lejeune ayant reçu des candidatures de religieuses enseignantes, dont celle de Marie, demande (a), dans sa Relation suivante une bâilleuse de fonds. Madeleine de La Peltrie, volontaire pour assumer cette charge, fait face à des empêchements majeurs (C, p. 904). Afin d'être aidée pour réaliser les démarches nécessaires, elle s'adjoint (b), en 1638, Bernières qui est orienté (c) sur Poncet qui informe (d) Marie qui rejoint (e) Madeleine de La Peltrie. 
Fig. 7 - Arrivée d'une bailleuse de fonds dans le réseau.

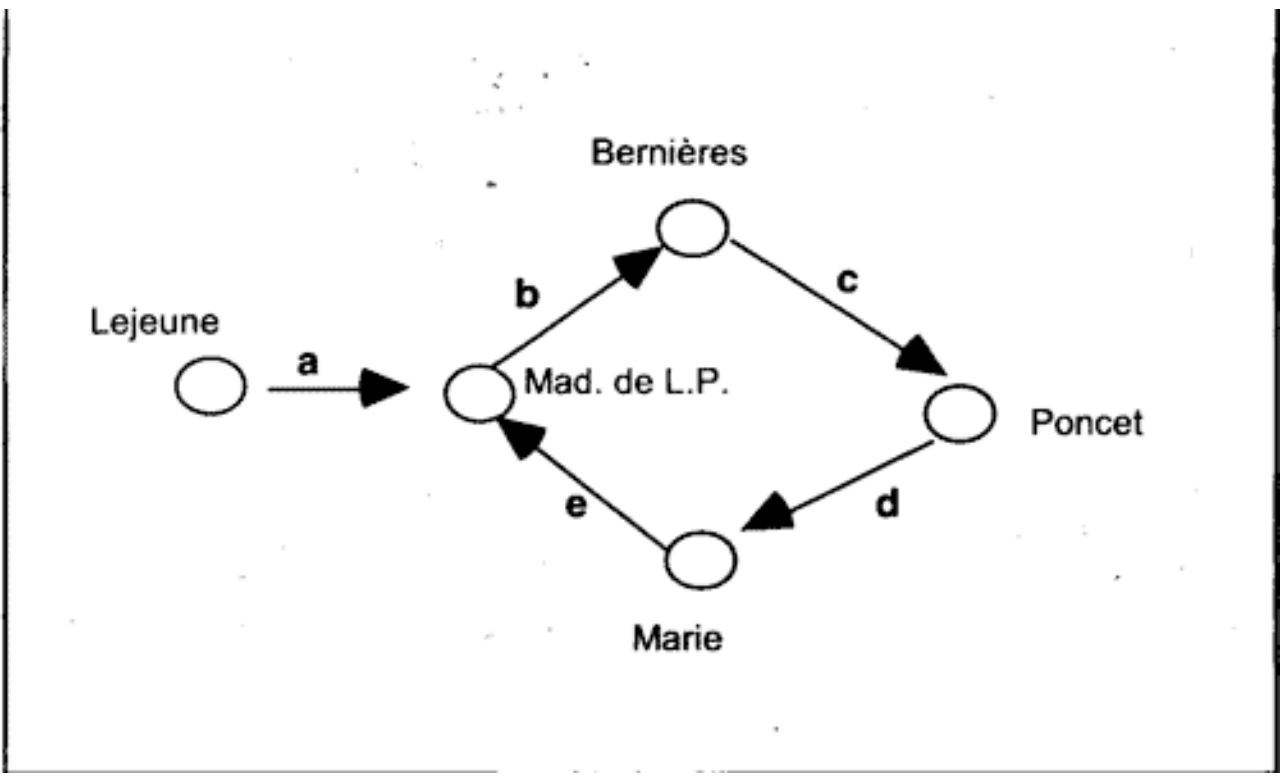

La mobilisation des autres accompagnatrices de Marie est aussi le fruit de la mobilisation de sous-réseaux précis et de démarches spécifiques du même type.

\section{Saturer d'informations une instance décisionnelle}

Saturer. Il ne s'agit pas d'envoyer des informations à tous vents, mais de faire converger celles qui sont pertinentes vers des têtes différentes de l'instance décisionnelle visée. C'est une stratégie de persuasion d'utilisateurs de réseaux qui consiste à renforcer les liens les uns par les autres en activant de façon concomitante des réseaux différents, mais qui doivent se concerter au sein d'une instance décisionnelle.

Pour partir en Nouvelle-France la candidature de Marie doit être acceptée par une instance politique et économique décisionnelle : la Compagnie des Cent-Associés. Celle-ci compte des représentants du pouvoir politique, des chapeliers, qui représentent l'aspect économique, des jésuites qui représentent Champlain et la mission. Ces blocs ne sont pas monolithiques et ne présentent pas forcément de convergences d'intérêts. Ainsi l'intérêt premier des chapeliers est d'être pourvus en peaux de castor et n'est pas l'établissement d'une école pour filles à Québec - réalité contestée en France même - qui pourrait retenir des colons dont ils ne désirent guère l'installation. Par ailleurs, la conversion des Amérindiennes n'est sans doute pas leur préoccupation primordiale. Tous les jésuites ne sont pas partisans de la mission de Nouvelle-France, Binet, provincial parisien jusqu'au début de 1639, n'est pas un mystique et se comporte en adversaire déclaré. Pour lui, il y a déjà assez à faire en France. Parmi les jésuites partisans de cette mission, Vimont, qui doit prendre la relève de Le Jeune en 1639, est opposé à la venue d'ursulines de Tours et préfère des parisiennes, plus proches des instances décisionnelles, d'une bonne noblesse de robe, plus riches et sans doute plus conventionnelles.

Lorsque le 11 janvier 1639 un débat a lieu parmi les Cent-Associés pour étudier le cas de Marie Guyart et des ursulines de Tours, financées par Madeleine de La Peltrie, la bataille est loin d'être gagnée d'avance. Quand bien même Madeleine serait autorisée à partir, puisqu'elle peut financer la fondation, le cas de Marie est fort délicat. On ne peut lui 
permettre de prendre le départ que s'il se trouve parmi les Cent-Associés plusieurs personnes issues de sphères différentes pouvant dire ce que l'on peut entendre dans certains comités de sélection d'aujourd'hui en lisant un curriculum vitae :

«I don't know her personnally, but several people whose opinion I trust have spoken well of her. « (Ronald S. Burt, 1992, p. 63)

Or en observant la figure 1 , on constate qu'elle rejoint les Cent-Associés par l'intermédiaire de La Haye, Dinet, du tandem La Peltrie-Bernières, de Raymond et de Sillery. Certaines informations puisées hors de ses lettres permettent de constater qu'elle a pu rejoindre aussi les Cent-Associés, peut-être sans le savoir, par des voies parallèles : Joseph-Antoine Poncet, fils d'un membre des Cent-Associés ${ }^{38}$; - Raymond dont le supérieur général, Charles de Lauson, est le propre frère de Jean de Lauson, administrateur de la Compagnie des Cent-Associés (O, p. 264 - V, p. 339.). Il est probable qu'il a parlé de Marie à son général qui a transmis le message à son frère ; - Charles de Saint-Etienne de la Tour, père d'une novice métis amérindienne entrée chez les ursulines de Tours en 1637, membre des Cent-Associés ${ }^{39}$; - des gens originaires de Tours, membres des Cent-Associés et qui connaissent peut-être Marie (les Quentin et Robin, Razilly). Ainsi, elle a pu faire «saturer » d'informations positives les Cent-Associés par, au moins, huit voies différentes (figure 8).

Lorsque le débat s'engage, les mystiques sont déjà persuadés, au moins par la filière de Raymond, sans oublier celles de Dinet et Poncet. Restent les opposants - les traditionalistes opposés à tout changement, les chapeliers, ceux qui pourraient préférer une parisienne ou une femme d'extraction moins modeste, noble ou plus riche et, non les moindres, les religieux non mystiques représentés par la tendance Binet.

C'est une plaidoirie du père de La Haye qui finit par convaincre (JII, p. 231). Avec quels arguments? Elle a toutes les qualités requises. Qualités que la majorité des assistants reconnait car ils connaissent quelqu'un qui l'a rencontrée ou qu'ils ont entendu dire du bien d'elle par des personnes en qui ils ont entière confiance. On peut observer que le réseau, activé aux endroits judicieux, a concentré et légitimé l'information sur Marie auprès des Cent-Associés et que cela s'est passé au bon moment. Tout se joue en une réunion des Cent-Associés le 11 janvier 1639. Les contacts devaient être antérieurs. 
Fig. 8 - Comment Marie Guyart rejoint les Cents-Associés.

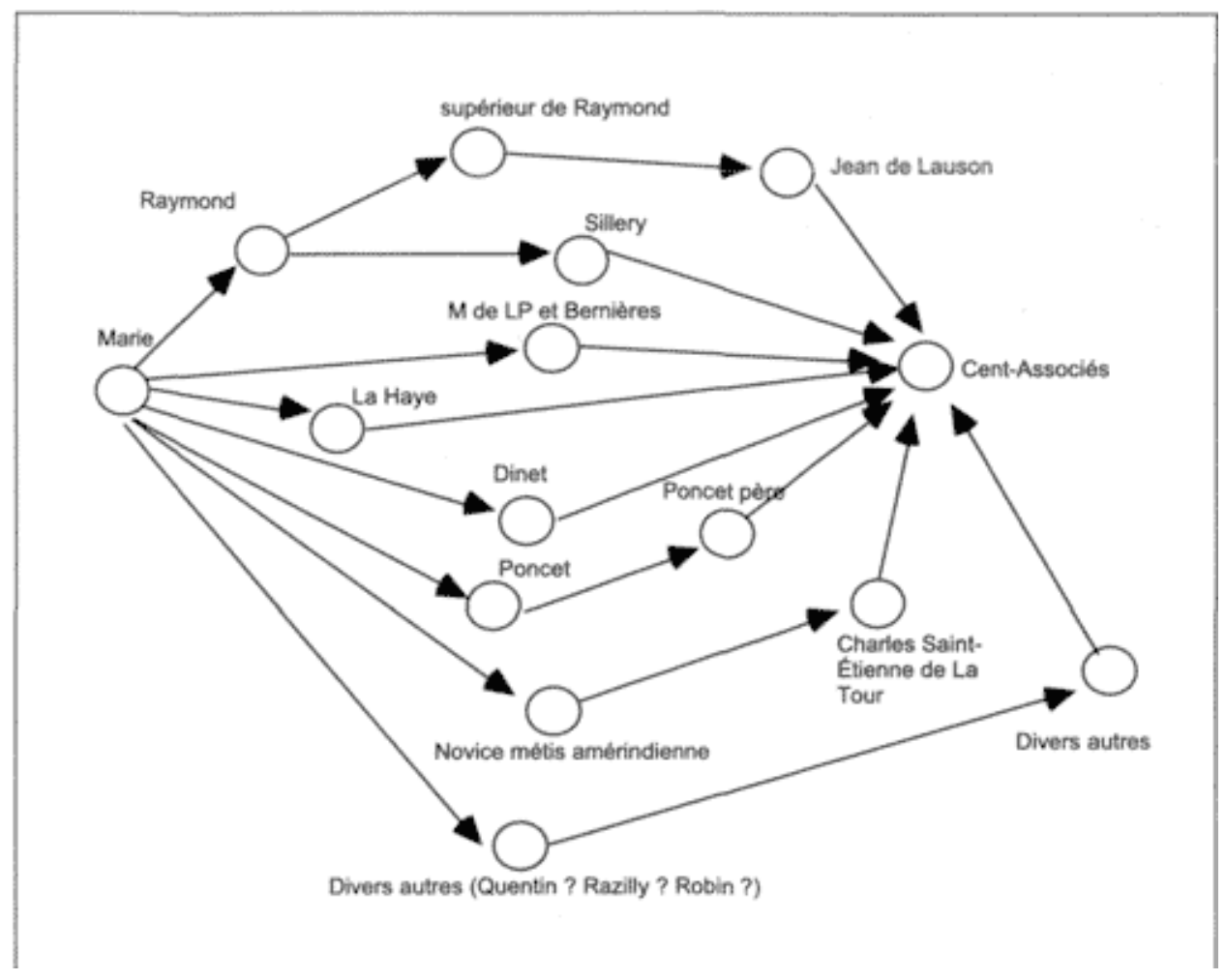

Il y a trois catégories de "saturateurs » dans le réseau : 1 - les gens que Marie connaît directement mais qui ne font pas partie des Cent-Associés; ils en connaissent des membres - ou des gens qui connaissent des membres - et peuvent la faire connaître, comme c'est le cas de Raymond et de Poncet ; 2 - les gens que Marie connaît directement et qui sont membres des Cent-Associés: La Haye, Dinet, peut-être Saint-Étienne de la Tour ; 3 - les gens que Marie ne connaît pas, mais qui sont (bien) avertis à son sujet par ceux de la première catégorie. C'est le cas du père de Poncet, mais aussi de Sillery, de Lauson ou d'amis des gens de la première catégorie que ni Marie ni nous ne pouvons identifier. Leur point de vue sur Marie est beaucoup plus efficace que si Marie s'était présentée elle-même pour vanter ses qualités ${ }^{40}$. Elle avait, le 11 janvier 1639, beaucoup mieux qu'elle-même, des contacts, dont le meilleur avocat qui pouvait galvaniser une opinion déjà « bien » informée : Georges de La Haye. Et elle n'a pas perdu le contact avec ses contacts. Elle a su garder ses liens actifs au cours du temps. Depuis le début de ses démarches, elle aurait pu négliger l'un ou l'autre. Ils ont gardé confiance. Certains liens se sont renforcés entre eux, car les gens se connaissaient par ailleurs : les trois jésuites, et, parmi eux, Dinet et Poncet, qui la connaissent par son fils.

\section{Utiliser des voies alternatives}

Utiliser des voies alternatives permet de pousser des ramifications très loin dans les milieux de pouvoir économique et politique qui peuvent rejaillir sur des hiérarchies très proches. De plus, en cas de défaillance d'une connexion, l'efficacité de la redondance d'autres relations permet de demeurer branché malgré un environnement changeant. Nous avons déjà montré qu'une personne importante, dont l'autorisation est la première nécessaire au projet, Françoise, a donné un avis favorable, l'a retiré et l'a réitéré lorsque 
l'avis favorable conditionnel de Lejeune et surtout la nouvelle de l'arrivée de la bâilleuse de fonds lui sont parvenus. Par ailleurs, l'évêque de Tours, réputé difficile, était redouté comme opposant au projet. Or il a accepté. Si l'on en croit Marie, dans sa seconde autobiographie de 1654, Françoise et l'archevêque de Tours n'auraient « jamais consenti » ni « donné obédience » sans l'influence de dom Raymond (JII, p. 221). Un réseau positif souterrain qu'on pourrait représenter ainsi a donc joué favorablement :

Fig. 9 - Une double voie alternative.

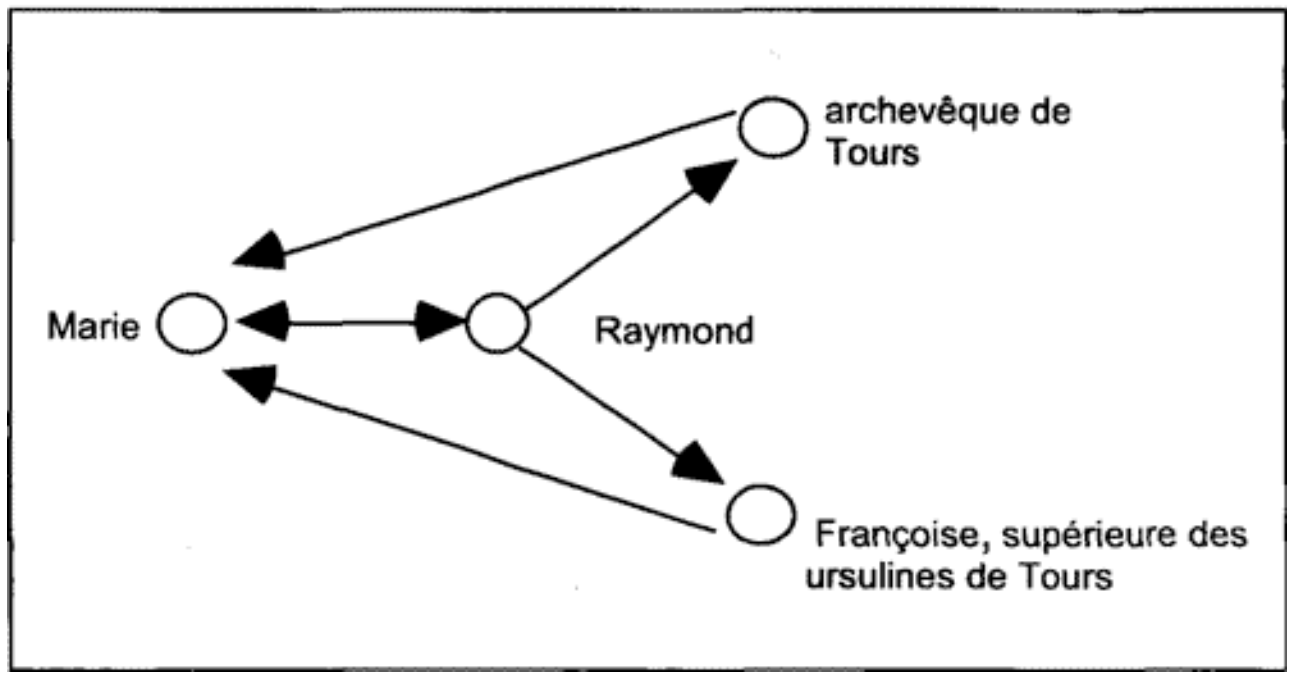

\section{Conclusion}

L'analyse de la mobilisation de ressources réalisée par Marie Guyart à travers des structures et des stratégies réticulaires nous a permis d'élaborer et d'illustrer plusieurs propositions analytiques qui pourraient se résumer ainsi : 1) En cas de disproportion entre le statut d'une personne et la difficulté de l'objectif visé, cet entrepreneur peut mobiliser des ressources s'il occupe une position-pivot, qui se situe à la jointure de plusieurs réseaux sociaux dont l'identité est antérieure à son action. 2) Pour identifier et choisir les personnes qui pourront fournir de l'information ou participer à un projet novateur et risqué, l'entrepreneur utilise sa compétence réticulaire pour repérer dans chaque personne rencontrée, au-delà du rôle qui motive cette première rencontre, d'autres rôles potentiels. 3) L'entrepreneur situé en bas de l'échelle sociale doit, pour pouvoir agir, être relié à certaines personnes par des liens forts et à d'autres, par des liens faibles. Ces derniers ne peuvent pas être utilisés immédiatement car la personne en question est elle-même en situation de faiblesse. 4) Il est nécessaire d'utiliser des personnes-relais, qui non seulement transmettent l'information sur le projet, mais jouent le rôle d'amplificateur de sa pertinence. 5) En cas de mobilisation de ressources à travers des réseaux sociaux, il convient de ne jouer la transparence qu'avec les personnes avec qui on est idéologiquement d'accord concernant le projet et de garder une grande discrétion (étanchéité) concernant les réseaux qui pourraient être opposés sur d'autres points. 6) Pour mobiliser des ressources à travers ses réseaux sociaux, une personne doit utiliser des stratégies diversifiées. Une stratégie spécifique, de type affinitaire, peut permettre de découvrir une personne rare, tandis qu'un lobbying intensif auprès d'instances décisionnelles peut finir par faire obtenir l'appui recherché. 
'approche par réseaux n'est fondée ni sur les structures, ni sur les acteurs isolés, mais sur les liens entre les acteurs dans et hors ces structures. Elle semble particulièrement pertinente pour l'étude de l'émergence sociale des mouvements religieux portés par un projet mystique. Elle permet l'analyse fine des stratégies socio-personnelles de mobilisation de ressources mises en œuvre pour la réalisation de ces projets. Leur aboutissement ne tient pas du miracle, mais d'opérations incarnées dans la "pâte " sociale.

\section{BIBLIOGRAPHIE}

Les sources sont référencées dans la note 11.

ADRIAZOLA Maria-Paul del Rosario, La connaissance spirituelle chez Marie de l'Incarnation, La «Thérèse de France et du Nouveau Monde ", Québec-Paris, Anne Sigier/Cerf, 1989.

ALDRICH H.E., MARSDEN P., “Environments and organizations" in N.J. SMELSER, ed., Handbook of Sociology, Beverly Hills Sage, 1988.

BERTRAND Dominique, La politique de saint Ignace de Loyola, Paris, Cerf, 1985.

BERTRAND Dominique, «Correspondance et pouvoir. Le réseau international de saint Ignace de Loyola » in Philippe DUJARDIN (textes réunis par) Du groupe au réseau, Lyon, C.N.R.S., 1988.

BERTRAND Dominique, «Dieu agit le premier, l'oraison de Marie de l'Incarnation » in Jean COMBY, éd., L'itinéraire mystique d'une femme, Marie de l'Incarnation, ursuline, Paris-Montréal, Cerf/ Bellarmin, 1993, pp. 101-131.

BERTRAND, Guy-Marie, « Expérience mystique de l'inhabitation - 3 - Marie de l'Incarnation » in Dictionnaire de spiritualité ascétique et mystique, tome VII - 2 - col. 1762-1767, 1971.

BIDART Claire, «L'amitié, les amis, leur histoire. Représentations et récits », Sociétés contemporaines, $\mathrm{n}^{\circ}$ 5, mars, L'Harmattan, Paris, 1991, pp. 21-42.

BOURDIEU Pierre, « Le capital social. Notes provisoires », Actes de la recherche en sciences sociales, 3, 1980, pp. 2-3.

BURT Ronald S., "The social structure of competition", in Nitin NOHRIA et Robert G. ECCLES, eds., Networks and Organizations, 1992, pp. 57-91.

BURT Ronald S., « Capital social et trous structuraux » (traduit par Emmanuel Lazega) in Revue française de sociologie (oct-déc, XXXVI - 4, 1995, pp. 599-628).

CANTER-KOHN Ruth, « Unité et multiplicité du réseau », in Pour, Le clair-obscur des réseaux, 1992, pp. 131-138.

CANTER-KOHN Ruth, "Le chemin, c'est le marcheur », Itinéraires de recherche, Perspectives documentaires en éducation, $n^{\circ} 28,1993$, pp. 27-46.

CHEVALIER Bernard, « Le milieu tourangeau au début du XVII : société et vie chrétienne », Colloque Marie de l'Incarnation -7-15, doc. poly., Ursulines de Tours, 1989. 
COLOMBEL Bernadette, « Marie de l'Incarnation ou une éthique du désir », Réseaux, n 64-65-66, 1992, pp. 35-45.

сомву Jean, éd., L'itinéraire mystique d'une femme, Marie de l'Incarnation, ursuline, Paris-Montréal, Cerf/Bellarmin, 1993.

CROZIER Michel, Erhard FRIEDBERG, L'acteur et le système, Paris, Le Seuil, 1977.

DEGENNE Alain, LEBEAUX M.O., "L'entraide entre les ménages : un facteur d'inégalité sociale ? ", Sociétés contemporaines, décembre, 8, 1991, pp. 21-42.

DEGENNE Alain, FORSÉ M., Les réseaux sociaux, Armand Colin, Paris, 1994.

DEROY Françoise, LÉOMANT Christian et PINEAU Gaston, éds, « Les réseaux sociaux, confrontation d'approches ", Études et Séminaires, Vaucresson, C.R.I.V./C.N.R.S. \& Laboratoire des Sciences de l'Éducation et de la Formation de l'Université François Rabelais de Tours, 1994.

DEROY-PINEAU Françoise, Madeleine de La Peltrie, amazone du Nouveau Monde, Montréal, Bellarmin, 1992.

DEROY-PINEAU Françoise, Marie de l'Incarnation. Marie Guyard femme d'affaires, mystique, mère de la Nouvelle-France 1599-1672, Montréal, Cerf/Bellarmin, 2000.

DESLANDRES Dominique, Le modèle français d'intégration socio-religieuse. Missions intérieures et premières missions canadiennes - 1600-1650, Thèse de doctorat (PhD), Université de Montréal, Département d'histoire, 2 tomes, 1990.

DESLANDRES Dominique, « Femmes missionnaires en Nouvelle-France. Les débuts des Ursulines et des Hospitalières à Québec » in Jean DELUMEAU, La religion de ma mère : Le rôle des femmes dans la transmission de la foi, Paris, Cerf, 1992, pp. 209-224.

DESLANDRES Dominique, « Le rayonnement des ursulines en Nouvelle-France » in C.E.R.C.O.R. Travaux et recherches, Les religieuses dans le cloître et dans le monde, Saint-Étienne, Publications de l’Université de Saint-Étienne, 1994, pp. 885-899.

FERRAND Alexis, Parents, habitants, citoyens. Meylan, banlieue grenobloise, C.N.R.S., Paris, 1982.

FERRAND Alexis, « La confidence : des relations au réseau » in Sociétés contemporaines, 5 mars, 1991, pp. 7-20.

FOISIL Madeleine, «L'époque moderne, XVI ${ }^{\mathrm{e}}$ et XVII ${ }^{\mathrm{e}}$ siècles » in Bernard PLONGERON, éd., Une histoire religieuse des origines de la Révolution, troisième partie, tome 1, Paris, C.N.R.S., 1987, pp. 215-306.

FORSÉ Michel, «Contribution à une morphologie des rôles réticulaires », in Sociétés contemporaines, 5 mars, 1991, pp. 43-53.

GERVAIS Pierre, Marie de l'Incarnation, Études de théologie spirituelle, Namur, Vie consacrée. 1996. GESTIN Marie-Louise, « Le style de Marie de l'Incarnation » in Jean Comby, éd., L'itinéraire mystique d'une femme, Marie de l'Incarnation, ursuline, Paris-Montréal. Cerf/Bellarmin, 1993, pp. 63-82. GoDBout Jacques-T. (avec la collaboration d'Alain Caillé). L'Esprit du don, Paris-Montréal, La Découverte-Boréal, 1992.

GRANOVETTER Mark, «The strength of weak ties », American Journal of Sociology, vol. 78, $\mathrm{n}^{\circ}$ 6, 1973, pp. 1360-1380.

GRANOVETTER Mark. Getting a Job. Cambridge (Mass.), Harvard University press, 1974. 
GRANOVETTER Mark, "Problems of explanation in economic sociology" in Nitin NOHRIA. Robert G. ECCLES, eds, Networks and Organization, 1992, pp. 25-56.

GUIART (textes réunis par Françoise Deroy-Pineau), Marie Guyard de l'Incarnation. Un destin transocéanique, Paris-Montréal, L'Harmattan, 2000.

GUIGUIÈRE Herman, « Une voix de l'indicible, le fond de l'âme », in Laval théologique et philosophique, juin 1997 (Raymond Brodeur, dir.), pp. 317-333.

GUIGUIÈRE Herman, « Les facettes du désir dans la lettre 68 de Marie de l'Incarnation » in Raymond BRODEUR, dir. Marie de l'Incarnation. Entre mère et fils : le dialogue des vocations, Québec, Presses de l'Université Laval, 2000, pp. 111-122.

IBARRA Herminia, « Structural alignments, individual strategies, and managerial action : elements toward a network theory of getting things done » in Nitin NOHRIA. Robert G. ECCLES, eds, Networks and Organization, 1992, pp. 165-188.

KAPFERER B.C., "Norms and the manipulations of relationship in a work context" in J.C. MITCHELL, ed., Social Networks in Urban Situations, Manchester, Manchester University Press, 1969.

KRACKHARDT David, "The strength of strong ties : the importance of philos in organizations" in Nitin NOHRIA, Robert G. ECCLES, eds. Networks and Organization, 1992, pp. 216-239.

KUMBASAR Ece, “Systematic Biases in Social Perception”, American Journal of Sociology, vol 100, tome 2, 1994, pp. 477-505.

LAZEGA Emmanuel, « Analyse de réseaux d'une organisation collégiale : les avocats d'affaires », Revue française de sociologie XXXIII (4), 1992, pp. 559-589.

LAZEGA Emmanuel, «Analyse de réseaux et sociologie des organisations », Revue française de sociologie, XXXV, 1994, pp. 293-320.

LAZEGA Emmanuel, « Présentation » d'Analyses de réseaux sociaux et structures relationnelles », Revue française de sociologie (oct-déc, XXXVI - 4), 1995a, pp. 593-597.

LAZEGA Emmanuel, (en collaboration avec Marie-Odile Lebeaux) «Capital social et contrainte latérale ", Revue française de sociologie (oct-déc, XXXVI - 4), 1995b, pp. 759-777.

LEMIEUX Vincent, À quoi servent les réseaux sociaux ?, Québec, éditions de l'I.Q.A.C, 2000.

LIN Nan, Social Structure and Network Analysis, Beverly Hills, Sage, 1982a.

LIN Nan, "Social resources and instrumental action", in Peter V. MARSDEN, Nan LIN, eds., Social Structure and Network Analysis, Beverly Hills, Sage, 1982b.

LIN Nan, «Les ressources sociales : une théorie du capital social », Revue française de sociologie (octdéc, XXXVI - 4), 1995, pp. 685-704.

MARSDEN Peter V., Lin Nan, eds.. Social Structure and Network Analysis, Beverly Hills, Sage, 1982.

MAUNIER René, « Recherches sur les échanges rituels en Afrique du Nord », Année sociologique (m.s.) II, 1927, pp. 12-97.

MAUSS Marcel (1923,1924, 1950), « Essai sur le don : forme et raison de l'échange dans les sociétés archaïques », Sociologie et anthropologie, Paris, Presses universitaires de France, 1968.

MICHEL Robert, o.m.i., Vivre dans l'Esprit : Marie de l'Incarnation, Montréal, Bellarmin, 1975.

MEEKS Wayne-A., The First Urban Christians - The Social Word of the Apostle Paul, New Haven-Londres, Yale University Press, 1983. 
NOHRIA Nitin, "The creation of new business ventures: the case of the 128 venture group" in Nitin NOHRIA, Robert G. ECCLES, eds. Networks and Organization, 1992, pp. 240-261.

NOHRIA Nitin, Robert G. ECCLES, eds. Networks and Organizations, Structure, Form and Action, Boston, Harvard business school press, 1992.

OURY dom Guy-Marie, o.s.b., « Marie de l'Incarnation », Québec, Les Presses de l'Université Laval/ Solesmes, abbaye Saint-Pierre, 1973.

OURY dom Guy-Marie, o.s.b., « Marie de l'Incarnation », Dictionnaire de spiritualité ascétique et mystique, tome X, 1980, col. 487-507.

RACINE Luc, Théorie de l'échange et circulation des produits sociaux, Montréal, Presses de l'Université de Montréal, 1979.

RACINE Luc, « Échange », in Pierre BONTE et M. IZARD, éds.. Dictionnaire de l'ethnologie et de l'anthropologie, Paris, Presses Universitaires de France, 1991a, pp. 211-213.

RACINE Luc, «L'obligation de rendre les présents et l'esprit de la chose donnée : de Marcel Mauss à René Maunier », Diogène, 154, 1991b, pp. 69-94.

REVUE FRANÇAISE DE SOCIOLOGIE, XXVI-4, octobre-décembre (études réunies et présentées par Emmanuel LAZEGA), Paris, Ophrys, 1995.

RIO Marie-Bénédicte, «La spiritualité ursuline au XVII ${ }^{\mathrm{e}}$ siècle : son apport à Marie de l'Incarnation », Colloque Marie de l'Incarnation, doc. poly.. Tours, Ursulines de Tours. 1989, pp. 25-39.

SIMMEL Georg (1917), Grundfagen der soziologie (Individuum und Gesellschaft), Berlin, G.J. Göschen. Traduction française, Sociologie et épistémologie, Paris, P.U.F., 1981.

SOCIÉTÉS CONTEMPORAINES, $\mathrm{n}^{\circ}$ 5, mars, (sous la direction d'Alain DEGENNE et Edmond PRETECEILLE) Réseaux sociaux, Paris, L'Harmattan, 1991.

TRUDEL Marcel, Histoire de la Nouvelle-France, volume III, La Seigneurie des Cent-Associés, 1627-1663, tome 1, Les Événements, Montréal. Bellarmin, 1979 et tome 2, La Société, Montréal, Bellarmin, 1983. VAN DER ELST, « L'économie du don et son éthique », in Humanisme et entreprise, n 55-56, février 2000, p. $77-88$.

WELLMAN Barry, “The community question: the intimate networks of east Yorkers", American journal of sociology, vol 84, n 5, 1979, pp. 1201-1231.

WELLMAN Barry, BERKowiTz S.D., eds, Social Structures. A Network Approach, Cambridge, Cambridge University Press, 1988a.

WELlman Barry, "The community question re-evaluated", in Michael Peter SMITH, "Power and the city", Comparative urban research, vol 1, New-Brunswick, (N.J.) Transaction, 1988 b.

Wellman Barry, CARRington P.J., HALl P.J., "Networks as personal communities" in Barry Wellman, S.D. BERKOWITZ, eds.. Social Structures. A Network Approach, Cambridge, Cambridge University Press, 1988a.

WELLMAN Barry, LEIGHTON B., « Réseau, quartier et communauté », Espace et société, 38-39, 1991, pp. 111-133. 


\section{NOTES}

1. À ne pas confondre avec Barbe Acarie qui entrera au Carmel sous le même nom après avoir introduit cet ordre en France.

2. Pendant la première moitié du XVII ${ }^{\mathrm{e}}$ siècle, un mouvement d'intégration socio-religieuse se développe pour faire accepter la "novellité ». Les femmes et les mystiques y sont très présents. Les missions intérieures et extérieures sont englobées dans ce vaste projet. «Pas une entreprise réformatrice qui ne soit initiée ... (sans) femmes » (DESLANDRES, 1990, p. 166); bien que leur présence soit souvent occultée. Le destin de Marie Guyart est exemplaire (DESLANDRES, 1992).

3. Voir notamment : - dans le Dictionnaire de spiritualité ascétique et mystique, les articles de dom OURY (1980) «Marie de l'Incarnation» et du père Guy-Marie BERTRAND (1971) «Expérience mystique de l'inhabitation »; - les ouvrages du père Robert MICHEL (1975) Vivre dans l'Esprit : Marie de l'Incarnation (1975), de Maria ADRIAzola (1989) La connaissance spirituelle chez Marie de l'Incarnation, La Thérèse de France et du Nouveau Monde, et de Pierre GERVAIS (1996) Marie de l'Incarnation, Études de théologie spirituelle. Lire également GIGUÈRE, 1977, 2000.

4. L'étonnement a notamment été souligné - outre les auteurs cités ci-dessus - par B. COLOMBEL (1992) et par J. сомву et al. (1993). Nous avons par ailleurs décrit le parcours de Marie Guyart ( DEROY-PINEAU, 2000).

5. Le thème du " moteur intérieur " a été emprunté à certains auteurs, notamment M.-L. GESTIN (1993, p. 75) et D. BERTRAND (1993, p. 125). Marie utilise aussi dans des sens équivalents les expressions: l'« Esprit qui me conduit», «Grand Dieu», "Amour ». L'expression "moteur intérieur » nous parle tout spécialement, car elle est dynamique et situe en une seule intériorité le Créateur et la volonté consentante et inventive de la créature. Il est bien clair que le «moteur» ne se réduit pas aux réseaux sociaux qui sont, en quelque sorte, ses courroies de transmission.

6. Les Relations des jésuites sont des lettres annuelles que ces religieux envoyaient de leurs missions à leurs confrères d'Europe pour les informer. Publiées en France de 1632 à 1672 et largement diffusées dans les milieux que l'on disait dévots, elles servaient aussi à convaincre leur public d'aider financièrement les missions. Elles demeurent une source fondamentale pour l'histoire de la Nouvelle-France et la connaissance des Amérindiens au XVII ${ }^{\mathrm{e}}$. En ce qui nous concerne, elles corroborent fréquemment la perception que Marie Guyart peut avoir des relations entre autruis.

7. Outre Jean de Bernières, nous rencontrerons dans cette aventure d'autres noms bien connus des milieux dévots de l'époque de Louis XIII : Lauzon, Sillery, les jésuites Dinet, Lalemant. Lejeune, des dames de la cour, la reine Anne d'Autriche elle-même. Les milieux dévots de Paris et de province sont actifs et fondent des missions. S'il y a au sein de ces milieux unanimité pour rendre service, bien des différends pouvaient exister sur la nature du service et sur la manière dont les femmes pouvaient s'y impliquer. La mission de Nouvelle France était très contestée par plusieurs (FOISIL, 1987 ; DESLANDRES, 1994).

8. Voir à ce sujet DESLANDRES (1994, p. 890).

9. Nous avons regroupé en trois les quatre catégories énumérées dans l'article de ALDRICH et MARSDEN (1988) : informations, ressources humaines, matières premières, capitaux. Déjà CROZIER et FRIEDBERG (1977) signalaient ces catégories de ressources dans L'acteur et le système.

10. Marcel MAUSS, R. MAUNIER et Claude LÉVI-STRAUSS continuent d'inspirer une tradition où se distingent notamment Jacques-T. GODBOUT et Alain CAILlÉ (1992); et Luc RACINE (1979,1991a, 1991b). Un récent article de Nicole VAN DERELST sur l'économie du don et son éthique actualise fort bien cette tradition (2000). 
11. Les œuvres de Marie Guyart fournissent le matériel de base de notre analyse. Il s'agit en particulier de la Correspondance (1971, références identifiées par le symbole C) éditée par dom Guy-Marie OURY, éditions Saint-Pierre de Solesmes, mais aussi des Écrits spirituels et historiques, publiés par dom Albert JAMET en 1928-1929, à partir d'une édition de dom Claude MARTIN, 2 vol., éditions Saint-Pierre de Solesmes, réédités en 1985 (comprenant deux autobiographies, 1633 et 1654, références identifiées respectivement par les symboles JI et JII) et La Vie de la Vénérable Mère Marie de l'Incarnation, 1677, par Claude MARTIN (reproduction en 1981 ) par les moines de Solesmes, références identifiées par le symbole V).

12. Le détail du raisonnement méthodologique est décrit dans une thèse de doctorat inédite soutenue à l'Université de Montréal (1996) et réalisée sous la direction de Luc RACINE et Paul BERNARD.

13. Nitin NOHRIA in "The Creation of New Business Ventures: the Case of the 128 Venture Group" (1992, p. 241).

14. Cette matrice est disponible sur demande auprès des auteurs.

15. Certains auteurs (par exemple Ece KUMBASAR, 1994) soulignent avec pertinence que les acteurs ont tendance à se percevoir une position centrale dans le réseau de relations dont ils font partie.

16. Précisons que si la majorité des lettres qui constituent la Correspondance est adressée à dom Claude Martin, fils de Marie Guyart, les lettres concernant la période que nous étudions sont essentiellement adressées à dom Raymond, un des meilleurs amis de Marie.

17. Essentiellement les Relations des jésuites et l'Histoire de la Nouvelle-France de Marcel TRUDEL, 1979, vol III, La seigneurie des Cent-Associés, tome I, Les Événements et tome II, La Société.

18. Voir notamment ce qu'en disent DEGENNE et FORSÉ (1994, p. 29). GRANOVETTER a abordé la question de la construction d'un réseau avec des données d'archives dans une communication à l' International sunhelt social network conference des 13-16 février 1997.

19. La thèse de Dominique DESLANDRES (1990, tome 2, pp. 384-385) sur les missions canadiennes controversées en France souligne cet aspect.

20. Pour une définition du capital social, nous nous référons à l'article bien connu de BOURDIEU (1980) « Le capital social. Notes provisoires».

21. Ronald S. BURT définit le «trou structural » comme un «tampon, comme un isolant dans un circuit électrique » $(1995$, p. 603). Les trous structuraux séparent des contacts et « procurent des bénéfices qui s'additionnent plus qu'ils ne se recouvrent » (id.)

22. Ce qui n'est pas sans rappeler la «triade interdite » de Mark GRANOVETTER (1973, p. 1362) que l'on envisagera plus bas.

23. LAZEGA traduisant Nan LIN (1995b, p. 687) rend embeddedness par insertion.

24. Rien ne prouve, en effet, que le jeune Poncet avait eu, contrairement à d'autres jésuites, d'autres manières de connaître Marie Guyart.

25. Ces travaux sont en cours de publication. On lira avec intérêt sur ce sujet deux articles de dom OURY « Sources et réédition des œuvres de Marie de l'Incarnation » et « La mystique nuptiale chez Marie de l'Incarnation », in Marie GUYART, 2000.

26. Terme chargé, même si ce mot, à l'époque, évoquait plus la faiblesse que l'idiotie; voir le Trésor de la langue française (CNRS-Gallimard, tome IX, 1981, p. 1158).

27. C'est-à-dire, entre autres, qu'elle bénéficie de toutes ses capacités mentales et d'une forte détermination.

28. Heureuse coïncidence pour Marie Guyart, c'est Poncet.

29. Proches des Cent-Associés et influents auprès d'eux. Ils ont une grande autorité.

30. Membres ou très proches des Cent-Associés.

31. S'il ne l'est pas encore le 11 janvier 1639, cela ne tardera pas. En tout cas, il est recteur du collège de Paris et va devenir incessamment provincial des jésuites de France (C, pp. 31-32). 
32. Pour reprendre une expression de Pierre CHAUNU dans sa préface à Dominique BERTRAND (1985, p. 8) qui forge le néologisme de sociodoxie : «...la sociologie (est) anachronique à cette époque, (on pourrait dire qu'il s'agit de) la manière dont la société globale est reçue, perçue, vécue par...»

33. Rappelons ce que le fils de Marie, dit de lui (c'est nous qui soulignons) : « Après (qu'il) eut fait la lecture de cette lettre... il ne l'approuva pas d'abord, le voyant (le dessein) si opposé à la condition d'une religieuse à qui la seule vue du monde doit faire peur et qui à plus forte raison doit avoir d'autres sentiments que... de faire des fonctions apostoliques dans un pays sauvage où il n'y avait pas même alors de l'assurance pour les hommes. » (V, p. 326)

34. Ce travail a été réactualisé par de nombreux auteurs. L'un des plus récents est LEMIEUX. 2000.

35. On ne développera pas ici la nature de l'utopie partagée. Il s'agit, bien entendu, du projet mystique de la Nouvelle-France dont les composantes, complexes, pourraient faire l'objet d'un autre travail.

36. Voir Dominique DESLANDRES (1990, tome 2, pp. 384-385).

37. Marie Guyart explique, dans sa relation de 1654, le rôle joué par les pères Dinet et de La Haye. Ils sont souvent venus à Tours. Le premier y a vécu et s'est occupé de son fils. Le second lui a conseillé d'écrire son autobiographie en 1633 (JI). L'un et l'autre la connaissent extrêmement bien, dans différentes facettes de sa vie.

38. TRUDEL, 1979, p. 419.

39. C : 61 - TRUDEL, 1979, p. 428.

40. BURT constate la même chose pour les entretiens d'embauche aujourd'hui (1992, pp. 57-91).

\section{RÉSUMÉS}

Marie Guyart (1599-1672) dite de l'Incarnation, Tourangelle sans argent ni pouvoir, mais poussée par son " moteur intérieur ", a réussi à passer en Nouvelle-France en 1639 pour mener à bien un projet qualifié d'insensé par son entourage : celui d'établir les ursulines à Québec afin d'assurer l'éducation des jeunes filles amérindiennes et françaises. Comment, de son cloître, a-t-elle réussi à transformer un projet mystique en réalisation sociale ? L'étude de la mobilisation de ressources permet de repérer l'utilisation stratégique de réseaux sociaux. L'approche par réseaux sociaux, déjà utilisée pour l'étude des projets de Paul de Tarse (Meeks, 1983) et d'Ignace de Loyola (Bertrand, 1985-1988), montre son intérêt pour la connaissance des rapports entre mystique et société. La mystique inspire des projets qui s'incarnent dans la société par des moyens tout à fait humains.

Marie Guyart (1599-1672), better known as Marie de l'Incarnation, was born in Tours, and did not share into significant money or power. And yet, propelled by her "interior motor", she succeeded in going to New France in 1639 to launch what most people around her considered an unrealistic project: establish a convent of the Ursulines in Quebec City, in order to teach French and Amerindian young girls. How did she succeed, from within her cloister, in transforming this mystical project into a social reality? We have studied how she strategically used social networks to mobilize the necessary resources. This social network approach had already proven useful for understanding the accomplishments of Paul from Tarse (Meeks, 1983), and of Ignatius of Loyola (Bertrand, 1985/1988); it has demonstrated its power in renewing the study of the links between 
mysticism and society. Mysticism inspires projects that find their expression in society using utterly human ways.

Marie Guyart (1599-1672), màs conocida como Marie de la Incarnación nació enTours, y no tenía ni dinero ni poder. Sin embargo, estimulada por su « fuerza motora interior » consiguió llegar a la Nueva-Francia en 1639 para conducir un proyecto que sus familiares percihieron como poco realista : establecer la orden de las Ursulinas en Québec a fin de permitir la educación de las muchachas ameriendas y francesas. De qué manera logró, desde su claustro. transformar este proyecto místico en verdadera realización social ? El estudio de la movilización de los recursos utiliza-dos resalta el uso estratégico de redes sociales. Este enfoque centrado en los redes sociales, que ya permitió entender cómo Pablo de Tarso (Meeks, 1983) y Ignacio de Loyola (Bertrand. 1985-1988) Ilevaron a cabo su proyectos, muestra aquí todo su inte-rés : renovar el estudio de las relaciones entre mística y sociedad. La mística inspira proyectos que se realizan en la sociedad por medio de recursos completamente humanos.

\section{AUTEURS}

\section{FRANÇOISE DEROY-PINEAU}

Département de sociologie, Université de Montréal

\section{PAUL BERNARD}

Département de sociologie, Université de Montréal 